\title{
Mitochondrial maturation drives germline stem cell differentiation in Caenorhabditis elegans
}

\author{
Nikolaos Charmpilas ${ }^{1,2} \cdot$ Nektarios Tavernarakis $\mathbb{1}^{1,3}$
}

Received: 23 November 2018 / Revised: 27 May 2019 / Accepted: 3 June 2019 / Published online: 19 June 2019

(c) The Author(s) 2019. This article is published with open access

\begin{abstract}
The C. elegans germline recapitulates mammalian stem cell niches and provides an effective platform for investigating key aspects of stem cell biology. However, the molecular and physiological requirements for germline stem cell homeostasis remain largely elusive. Here, we report that mitochondrial biogenesis and function are crucial for germline stem cell identity. We show that general transcription activity in germline mitochondria is highly compartmentalized, and determines mitochondrial maturation. RPOM-1, the mitochondrial RNA polymerase, is differentially expressed as germ nuclei progress from the distal to the proximal gonad arm to form oocytes. Mitochondria undergo changes from globular to tubular morphology and become polarized, as they approach the proximal gonad arm. Notably, this mitochondrial maturation trajectory is evolutionarily conserved. We find that a similar transition and temporal mitochondrial RNA polymerase expression profile characterizes differentiation of mammalian stem cells. In C. elegans, ATP, and ROS production increases sharply during maturation. Impaired mitochondrial bioenergetics causes gonad syncytium tumor formation by disrupting the balance between mitosis and differentiation to oocytes, which results in a marked reduction of fecundity. Consequently, compensatory apoptosis is induced in the germline. Sperm-derived signals promote mitochondrial maturation and proper germ cell differentiation via the MEK/ERK kinase pathway. Germ cell fate decisions are determined by a crosstalk between Insulin/IGF-1 and TGF- $\beta$ signaling, mitochondria and protein synthesis. Our findings demonstrate that mitochondrial transcription activity determines a shift in mitochondrial bioenergetics, which in turn regulates germline stem cell survival and differentiation. Perturbation of mitochondrial transcription hinders proper germ cell differentiation and causes germline tumor development.
\end{abstract}

\section{Introduction}

In adult Caenorhabditis elegans animals, somatic cells are postmitotic and terminally differentiated. Yet, adult

Edited by N. Chandel

Supplementary information The online version of this article (https:// doi.org/10.1038/s41418-019-0375-9) contains supplementary material, which is available to authorized users.

Nektarios Tavernarakis

tavernarakis@imbb.forth.gr

1 Institute of Molecular Biology and Biotechnology, Foundation for Research and Technology-Hellas, Heraklion, Greece

2 Department of Biology, University of Crete, Heraklion, Greece

3 Department of Basic Sciences, School of Medicine, University of Crete, 70013 Heraklion, Crete, Greece hermaphrodite nematodes possess a germ cell population undergoing rapid proliferation. Germ cells are topologically isolated from surrounding somatic tissues and are enclosed in two $U$-shaped gonads. At the distal tip each gonad, the distal tip cell (DTC), a somatic cell of mesenchymal origin, preserves the mitotic identity of nearby germ cell nuclei through GLP-1 (Notch)/LAG-2 (Delta) signaling. The DTC forms a plexus that surrounds adjacent nuclei [1]. The majority of germline nuclei divide once within the proliferative region [2]. Upon escaping from DTC's vicinity, they invariably progress toward meiosis I (pachytene, diplotene, and diakinesis), become enclosed by a cell membrane after the gonad turn and ultimately form oocytes, which are fertilized upon reaching the spermatheca in the proximal arm [3, 4]. The $C$. elegans germline shares several features analogous to mammalian stem cell niches; thus, providing an effective platform toward the delineation of cellular and molecular mechanisms underlying stem cell fate [5]. 
A distinguishing characteristic of stem cells relates to energy metabolism and mitochondrial function (reviewed in [6]). Mitochondria originate from endosymbiotic events of ancestral eukaryotic cells with once free-living proteobacteria [7]. Albeit they principally rely on nuclear gene products for their function, they are considered semiautonomous, since they retain their own genome (mtDNA). mtDNA mutations have been associated with diverse human diseases [8]. The human mtDNA encodes 13 electron transport chain (ETC) components, as well as two rRNAs (12S and $16 \mathrm{~S}$ rRNAs) and the whole repertoire of tRNA molecules [9]. These untranslated RNA species, together with exclusively nuclear-encoded mitochondrial ribosomal proteins, form the $55 \mathrm{~S}$ mitochondrial ribosome, which synthesizes mtDNA-derived ETC components in the organelle [10]. Transcription of the mitochondrial genome is mediated by a tripartite complex, comprising a dedicated RNA polymerase and two auxiliary transcription factors, TFAM and TFB2M [11-14].

Although mitochondria have been implicated in the maintenance of stemness and the progression toward differentiation, the contribution of the mitochondrial genome remains elusive. To gain relevant insight, we examined the involvement of mitochondrial genome expression in C. elegans germ cell homeostasis. We find that perturbations in general mitochondrial transcription and mitochondrial bioenergetics reduces fecundity and causes sterility. Depletion of RPOM-1, the homolog of the mammalian mitochondrial RNA polymerase, generates a tumor phenotype in the pachytene gonad region due to impaired germ cell differentiation, and leads to gonad collapse at elevated temperatures. Induction of apoptosis acts in a protective, compensatory manner to restrict tumor size. Gonadal mitochondrial biogenesis is coupled with extrinsic Insulin/IGF-1 and TGF- $\beta$ signaling pathways that converge on the germline. Attenuation of general protein synthesis in the germline alleviates the sequelae of RPOM-1 deficiency. Expression of mitochondrial RNA polymerase gradually increases in the course of germ cell differentiation, concurrent with morphological and functional adaptations, associated with enhanced metabolic activity of germline mitochondria. These adaptations are accompanied by increased ATP and ROS production in the proximal gonad arm. Notably, upregulation of mitochondrial RNA polymerase expression, the consequent establishment of tubular mitochondrial morphology and associated bioenergetic profile are evolutionarily conserved during mouse stem cell differentiation. Thus, coordinated expression of the mitochondrial genome is a key component of a regulatory program that determines germ cell differentiation.

\section{Materials and methods}

\section{Strains and genetics}

We followed standard procedures for maintaining C. elegans strains. Rearing temperature was set at $20^{\circ} \mathrm{C}$ for most of our experiments unless noted otherwise, but was raised at $25^{\circ} \mathrm{C}$ when germline expression was assessed to avoid transgene silencing that occurs at lower temperatures. The following nematode strains, which are available from CGC, were utilized in this study: N2: wild-type Bristol isolate, CU5991: $f z o-$ 1(tm1133) II, CU6372: drp-1(1108) IV, and DA631: eat-3 (ad426) II; and him-8(e1489) IV, MT7686: ced-9(n2812)/ qC1 [dpy-19(e1259) glp-1(q339)] III, MT1522: ced-3(n717) IV, CB1370: daf-2(e1370) III, DR40: daf-1(m40) IV, RB1206: rsks-1(ok1255) III, KX80: ife-5(ok1934) II, and CB4037: glp-1(e2141ts) III. We used the JJ1850: unc-119 (ed3) III; Is[his-72(1kb 5'UTR)::his-72::SRPVAT::GFP::his-

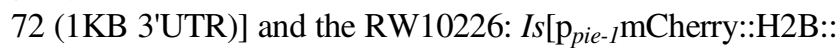
pie-1 3'UTR]; Is[phis-72HIS-24::mCherry::let-858 3'UTR] strains for monitoring germline nuclei in vivo, the AD189:

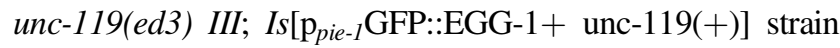
for monitoring oocyte membranes in vivo and the OD95: unc-

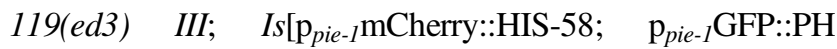
(PLC1delta1) +unc-119(+)] strain for simultaneously monitoring oocyte membranes and germline nuclei in vivo. JK4472: WT; Is $\left[\mathrm{p}_{\text {lag-2 }} \mathrm{MYR}::\right.$ tdTomato $\left.+\mathrm{p}_{t t x_{-3}} \mathrm{GFP}\right]$ and JK4475: WT; Is $\left[\mathrm{p}_{\text {lag-2 } 2} \mathrm{MYR}:: \mathrm{GFP}+\mathrm{p}_{t t x-3}\right.$ DsRed] were used for monitoring the DTC's membrane plexus, while DG1575: $\mathrm{WT}$; Is $\mathrm{p}_{\text {lim- }}$ - GFP + rol-6(su1006)] was used for monitoring gonad sheath morphology. All the experiments were performed using day 1 (D1) gravid adult animals that were precisely synchronized at the L4 larval stage one day earlier. For $g l p-1(e 2141)$ mutants specifically, we placed eggs on plates at the restrictive temperature of $25^{\circ} \mathrm{C}$ for $48 \mathrm{~h}$. Then we transferred L4 larvae on fresh control or rpom-1(RNAi) plates and we placed them for one more day at $20^{\circ} \mathrm{C}$ prior to nuclear staining and microscopic observation. Culture of $g l p$ 1(e2141) mutants at $25^{\circ} \mathrm{C}$ for $48 \mathrm{~h}$ during this developmental window renders $g l p-1(e 2141)$ mutants completely sterile [15]. The following strains were generated in the current study: IR1677: WT; Ex[p CEOP $1740_{0} \mathrm{RPOM}-1:: \mathrm{GFP}:$ :unc-54 3'UTR],

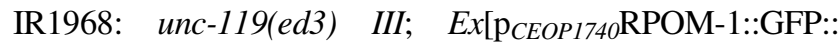
rpom-1 3'UTR + unc-119(+)], IR1966: unc-119(ed3) III; and Is $\left[\mathrm{p}_{\text {pie-1 }}\right.$ Perceval::tbb-2 3'UTR +unc-119(+)].

\section{Molecular cloning}

Gene inactivation was achieved by bacterial feeding of $C$. elegans with RNAi clones expressing double-stranded RNA targeting the gene of interest. All RNAi treatments were performed continuously from hatching (L1 larval stage) till the day of observation (D1). The following primers were 
used for generation of RNAi constructs used in the present study. For rpom-1(RNAi): 5'-ATGAGAAGACTGGAACG AATTGTC- $3^{\prime}$ and 5'-TAGTGTTCAATCCCTCACCAAT C-3', for $h m g-5(R N A i)$ : 5'-GGATCCAATGTTGGGAACA ATTTC-3' and 5'-ACCGGTGGTTGATCTGCATTTT C-3', for $t f b m-1(R N A i)$ : 5'-ATGGCTTCTGCTTCACGTCT CC-3' and 5'-CATCTTAGGCTCTGCCACGTATTG-3', for $g l d-1(R N A i)$ : 5'-CACTCCAACTTACGGTGTTTCG-3' and $5^{\prime}$-TCTACCGACGAAGTTATACTGAAATG-3', for $m p k-1(R N A i): \quad$ FW: $5^{\prime}$-GTTCATGGGCAACTTTTTGA G-3' and 5'-GATTACTGAGCATTTCTGCGAG-3', for $m e k-2(R N A i)$ : 5'-CGTAATCCGTTGGGACTCAG-3' and 5'-CGAGAATCCTGCGAGAACTG-3', for goa-1(RNAi): 5'-CCACATACAGTGAGTGAGTAGAG-3' and 5'-CTCT CTGTCAGCCGAACC-3', and for $g s a-1(R N A i)$ : $5^{\prime}$-AGCA AAAAAGAACGAGCAAC-3' and 5'-TGTCCTCCCAGA GTACAAGA-3'.

For cloning the minimal $2.1 \mathrm{~kb}$ operon promoter upstream of CEOP1740 operon, which contains the rpom-1 gene, we used the 5'-CCATGAAATTGAGGATTCTGA AAC- ${ }^{\prime}$ and 5' - ATTTCCGTTTAGTAGCGATTTTTAAC AG-3' primer pair. The corresponding product was inserted in TOPO and then in pPD95.77 linearized with PstI-BamHI digestion. For cloning the $3.5 \mathrm{~kb}$ rpom- 1 full length cDNA, we utilized $C$. elegans total RNA as a template to synthesize the rpom- 1 single-strand cDNA with the PrimeScript ${ }^{\mathrm{TM}}$ Reverse Transcriptase kit (Taqara) and the 5'ACCGGTGGACTAAAAAAATAAACAGAATCCTTGA $\mathrm{C}-3^{\prime}$ gene-specific reverse primer. Then, the previous primer was used in the same reaction with the 5'-GGATCCA TGAGAAGACTGGAACGAATTGTC- $3^{\prime}$ forward primer to synthesize the full length rpom-1 cDNA. Expand highfidelity DNA polymerase (Roche) was used in the PCR reaction to minimize errors. The amplified fragment was inserted upstream of GFP in the pPD95.77 expression vector linearized with BamHI-AgeI restriction digestion. For amplifying the $0.1 \mathrm{~kb}$ rpom-1 3'UTR fragment, we used the 5'-GAATTCAGTTAGAAGTGTTTTTTTTGTTG-3' and 5'-GGGCCCCATTTTCTGATTCCAGCG-3' pair of primers. Upon ligation into TOPO vector, we isolated the corresponding fragment using EcoRI-ApaI restriction digestion. This was then inserted in pPD95.77 linearized with EcoRI-ApaI digestion downstream of the rpom-1 cDNA::GFP cassette to replace the unc-54 3'UTR fragment. For creating the pie-1 promoter Perceval::tbb-2 3'UTR plasmid, we extracted the Perceval from the pRsetB-his7Perceval plasmid (catalog number 20336, Addgene) using Xbal-XhoI digestion. In parallel, we amplified the tbb-2 3' UTR using the 5'-CTCGAGCAATAAATGCAAGATC

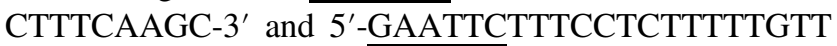
GGGTCACTC-3' pair of primers. We digested pPD95.77 with XbaI-EcoRI digestion to remove GFP and we simultaneously ligated the two inserts with the linearized vector in a single reaction. An extended pie-1 promoter was amplified using the 5'-GTCGACTCGTATTTCTCAGTCA TTTTTGTG- $3^{\prime}$ and $5^{\prime}$-CCATGGATCGTTTTGTATTCT GTGTGCTGG-3' primer pair and was inserted upstream of the Perceval::tbb-2 cassette using SalI-NcoI restriction sites.

\section{Nematode strain generation}

We used both microinjection into $C$. elegans gonads and bombardment with gold nanoparticles to generate transgenic strains. To avoid undesired transgene silencing in the germline, in microinjections we used low micromolar concentrations $(5 \mathrm{ng} / \mu \mathrm{L})$ of both the reporter plasmid and the cotransformation marker and an excess of PvuII-digested $E$. coli genomic fragments $(50 \mathrm{ng} / \mu \mathrm{L})$, as previously described [16].

\section{Mitochondrial DNA quantification}

mtDNA was quantified by quantitative real-time PCR-based method as previously described [17]. The 5'-GTTTATG CTGCTGTAGCGTG-3' and 5'-CTGTTAAAGCAAGTGG ACGAG-3' primer pair was used for measuring mtDNA levels. These primers hybridize in the atp- 6 gene which is located in the mitochondrial genome (mtDNA). The results were normalized to genomic DNA amplified with the $5^{\prime}$ TGGAACTCTGGAGTCACACC-3' and 5'-CATCCTCCT TCATTGAACGG-3' primer pair, which hybridizes to the genomic region of ama-1 gene. Quantitative PCR was performed using a Bio-Rad CFX96 real-time PCR system, and was repeated three times.

\section{Measurements of mitochondrial activity}

Staining with mitochondrial dyes (TMRE, Mitotracker ROS) to assess functional mitochondria and ATP measurements in whole animals were performed as previously described [18].

\section{mRNA quantification}

Total RNA from synchronized D4 animals was extracted using the TRIzol reagent (Invitrogen). The following sets of real-time primers were used in the current study: for measuring hmg-5 mRNA levels: FW: 5'-CGTCCAAGTGT TCCTCCAAGTG-3' and 5'-CTTCGCTTCGTCTGTGTA CTTCTTT- $3^{\prime}$, for measuring $t f b m-1$ mRNA levels: $5^{\prime}$-CAC AAGAAAGATAGCAAAACACGC- ${ }^{\prime}$ and $5^{\prime}$-CGAGATG CTGTAACGGCGG- $3^{\prime}$, and for measuring rpom- 1 mRNA levels: 5'-GGTGTCGGCTGGTATCCTCAAC-3' and 5'-T GGCACAATCTCCTGAGTAGCC-3'. 


\section{Egg laying assays}

For assessing egg laying, we placed single, precisely synchronized gravid adult nematodes (at D1-D2 of adulthood) in individual $33 \mathrm{~mm}$ NGM plates seeded with a standard OP50 bacterial lawn. The number of eggs that each adult worm produced in the next $12 \mathrm{~h}$ were measured. At least 20 individual measurements were acquired per experimental condition. The egg laying assays were performed at $20^{\circ} \mathrm{C}$, apart from the one corresponding to the mitochondrial dynamics mutants (fzo-1, drp-1, and eat-3) that were performed at $25^{\circ} \mathrm{C}$.

\section{Antimycin A treatment}

Antimycin A (catalogue number A8674, Sigma-Aldrich) was supplemented on top of NGM plates seeded with OP50 E. coli bacterial food at a final concentration of $50 \mathrm{nM}$ per plate. The plates were previously placed in a UVcrosslinker for $15 \mathrm{~min}$ to kill off bacteria before Antimycin A addition. L4-staged nematodes were placed on Antimycin A-containing plates for 2 days prior to microscopic observation. The entire experiment was performed at $25^{\circ} \mathrm{C}$ to avoid undesired silencing of the Percevalexpressing transgene.

\section{Nuclear staining of nematodes}

We synchronized adult worms of the genotypes of interest at the first day of adulthood (D1). We stained with Hoechst 33342 to monitor germline nuclei at this stage. First we washed NGM plates with M9 buffer and collected the animals in a $1.5 \mathrm{~mL}$ Eppendorf tube. We let the worms pellet with gravity and we washed once with PBSTween $20^{\circ} 0.01 \%$. We centrifuged at $3000 \mathrm{rpm}$ for $1 \mathrm{~min}$ to pellet the animals and we removed the supernatant. Fixation was performed with cold methanol $100 \%$ for maximum $5 \mathrm{~min}$ at $-20^{\circ} \mathrm{C}$. The Eppendorf tubes were spinned and the fixative was removed. Upon fixation, cell membranes were permeated by washing once with PBSTween $20^{\circ} 0.1 \%$. Upon centrifugation at $3000 \mathrm{rpm}$ for $1 \mathrm{~min}$, the worm pellet was stained by adding $300 \mu \mathrm{L}$ of diluted Hoechst 33342 solution (final concentration $1 \mu \mathrm{g} / \mathrm{mL}$ ) for $5 \mathrm{~min}$ in the dark. The Eppendorf tubes were centrifuged, the supernatant was removed and the pellet was washed for a final time with PBS-Tween $20^{\circ} 0.1 \%$ to remove excessive Hoechst stain. The supernatant was aspirated; $20 \mu \mathrm{L} 80 \%$ glycerol was added in each Eppendorf tube and the samples were mounted in microscopic slides prior to observation. The transition zone nuclei were distinguished due to the polarized localization of chromosomes in germ nuclei [19].

\section{Immunofluorescence of $C$. elegans gonads}

A large number of synchronized D1 adult worms grown on NGM plates were washed two repetitive times with M9 buffer and $20 \mu \mathrm{L}$ of $20 \mathrm{mM}$ levamisole was added on the worm pellet to anaesthetize the animals. About $7-8 \mu \mathrm{L}$ of the mixture was placed on top of polylysine-treated slides and the animals were dissected using a $8 \mathrm{~mm}$ insulin syringe, until the gonads were released from the animals due to mechanical pressure. The extracted tissue was fixed with 4\% paraformaldehyde in PBS for $20 \mathrm{~min}$ at room temperature. The fixative was removed by washing the specimen twice with PBS. Then, the gonads were permeabilized with $0.2 \%$ Triton X-100 in PBS $1 \times$ for 5 min and rinsed twice with PBS. Next, the tissue was incubated in blocking solution (1\% BSA in PBS-Tween 0.02\%) for $1 \mathrm{~h}$ at room temperature. The incubation with the rabbit anti-phosphoHistone H3 (Serine 10) (06-570, Sigma-Aldrich) antibody (diluted 1:300 in blocking solution) was performed overnight in the dark at $4{ }^{\circ} \mathrm{C}$ and the slides were sealed to avoid evaporation. The sample was washed thrice with PBSTween $0.02 \%$ to remove the primary antibody excess. Finally, the gonads were incubated for $1 \mathrm{~h}$ with the antirabbit IgG AlexaFluor 488 (catalogue number ab150077, Abcam) secondary antibody (diluted 1:500 in PBS-Tween $0.02 \%$ ) and DAPI (final concentration $2 \mu \mathrm{g} / \mathrm{mL}$ ) to stain germline nuclei, rinsed three times with PBS and mounted with glycerol prior to observation.

\section{Staining germline mitochondria in live animals}

To stain germline mitochondria, mitochondrial dyes were added on top of standard NGM plates seeded with OP50 $E$. coli bacterial food. The plates were previously placed in a UV-crosslinker for $15 \mathrm{~min}$ to kill off bacteria and avoid undesired catabolism of compounds. TMRE (Tetramethylrhodamine, ethyl ester, perchlorate, catalog number T-669; Molecular Probes, Invitrogen) and Mitotracker Red CM-H2X ROS (catalog number M-7513; Molecular Probes, Invitrogen) were administered by food in a final concentration of $1 \mu \mathrm{M}$ per plate and DIOC6(3) (3,3'-Dihexyloxacarbocyanine Iodide, CAS Number 53213-82-4, Sigma-Aldrich) in a final concentration of $2 \mu \mathrm{M}$ per plate. The plates were allowed to dry, constantly protected from light, and then wild-type eggs harvested from hypochloritetreated, well-fed adult animals were placed on top of the bacterial lawn. The larvae that hatched were fed with the dyes for three consecutive days at $25^{\circ} \mathrm{C}$ (when His-72::GFP transgenic animals were used) or for four consecutive days at $20{ }^{\circ} \mathrm{C}$ (when wild-type animals were used), until they produced D1-2 gravid adult nematodes. Adult animals were anaesthetized with $20 \mathrm{mM}$ levamisole and were 
microscopically examined using a ZEISS LSM 710 confocal microscope.

\section{Mammalian cell culture and immunofluorescence}

Standard cell culture procedures were followed. J1 embryonic stem cells were initially cultured for four passages on top of Mitomycin C-treated mouse fibroblasts and then on gelatin in LIF cytokine-containing ES medium to preserve their pluripotent identity. Large globular ES colonies were acquired. LIF was removed and the ES medium was replaced with EB medium to favor unbiased differentiation toward multiple cell lineages. Cells were grown for up to $48 \mathrm{~h}$ on EB medium on 12-well plates on top of cover slips. For immunofluorescence experiments, cells were washed three times with PBS before they were fixed with $4 \%$ paraformaldehyde in RT. The cover slips were washed again three times with PBS. The blocking was performed with $0.2 \%$ Triton $\mathrm{X}, 10 \% \mathrm{FBS}$ for $1 \mathrm{~h}$. Mouse monoclonal anti-MTCO1 (catalogue number ab14705, Abcam), rabbit polyclonal anti-POLRMT (catalogue number PA5-28196, Thermo Scientific), and mouse monoclonal anti-OCT4 (catalogue number sc-5279, Santa Cruz Biotechnology) were used as primary antibodies according to the manufacturer's instructions. The appropriate primary antibody combination was added in blocking solution and was incubated overnight with the specimen at $4{ }^{\circ} \mathrm{C}$. Next day, the specimen was rinsed thrice with $\mathrm{PBS}$ and was incubated with anti-rabbit IgG AlexaFluor 488 (catalogue number ab150077, Abcam), anti-mouse IgG AlexaFluor 594 (catalogue number ab150116, Abcam) fluorescent secondary antibodies and Hoechst 33342 diluted in PBS for an hour in RT. Finally, the slides were mounted with prolong ${ }^{\circledast}$ gold antifade reagent (catalogue number 9071, cell signaling) and stored, protected from light at $4{ }^{\circ} \mathrm{C}$ prior to microscopic observation.

\section{Results}

\section{Intact mitochondrial function is indispensable for fecundity in C. elegans}

The mitochondrial genome of $C$. elegans encodes 12 ETC protein-coding genes lacking the ATP8 gene of its human counterpart [20]. The following proteins have been proposed to engage in mtDNA metabolism: HMG-5, a putative nematode homolog to the mammalian TFAM, is a high-mobility group protein that regulates mitochondrial DNA content and has important roles in replication, transcription, and packaging of mtDNA into nucleoids [21, 22]. C. elegans genome encodes a TFB1M homolog, a protein with $16 \mathrm{~S}$ rRNA methyltransferase activity, which shares some properties, but has distinct functions from TFB2M [23]. Rpom-1 gene encodes the mammalian POLRMT homolog RPOM-1. Alignment of the catalytic domain of RPOM-1 and its putative homologs revealed extensive conservation from yeast to mammals (Fig. S1). We created RNAi constructs to specifically target $h m g-5$, rpom- 1 and tfbm- 1 transcripts (Fig. S2A), since mutants for the respective genes are lethal and sterile. We found that RNAi-mediated knockdown of hmg-5, rpom-1, and $t f b m-1$ compromised mitochondrial function, as evidenced by a decrease in TMRE (Tetramethylrhodamine, ethyl ester, perchlorate) and MitoTracker Red CMH2XROS stainings, as well as a reduction in ATP levels (Fig. S2B-D). Importantly, RPOM-1 depletion dramatically reduced mtDNA content, at levels comparable to HMG-5 depletion (Fig. S2E). This can be attributed to the fact that POLRMTs also provide RNA primers for the initiation of mtDNA replication by the mitochondrialspecific DNA polymerase. Taken together, these results highlight the importance of intact transcription machinery residing in mitochondria for preserving full organelle function.

We noticed that rpom-1 knockdown dramatically reduced the brood size of wild-type nematodes (Fig. 1a, b). The defect was even more pronounced in subsequent generations, indicating the existence of a maternal-effect phenotype (Fig. 1c). Intriguingly, we detected the formation of germline tumors in the pachytene syncytium region of animals fed with bacteria expressing rpom-1 (RNAi) (Fig. 1d). We reasoned that this phenotype could be a consequence of elevated mitotic activity in the proliferative region of the gonad. To test this hypothesis, we stained extruded gonads from control and rpom-1(RNAi)treated animals with an antibody specific to phosphorylated serine 10 of histone $\mathrm{H} 3$ (anti-pH3), a widely-used marker for mitotic-phase nuclei [24, 25]. RPOM-1 depletion did not cause any significant change in the number of mitotic nuclei (Fig. 1e, f). We then investigated whether germ nuclei accumulate due to their inability to properly differentiate and produce oocytes. By utilizing a fluorescent reporter strain for EGG-1, a protein that localizes to oocyte membranes, we detected three times less EGG-1::GFP positive oocytes in rpom-1 (RNAi)-treated animals compared to their control counterparts, as well as less nuclei in diplotene and diakinesis (Fig. 1g-i). Furthermore, we did not observe any gross morphological defect in somatic tissues that support the gonads, such as the gonad sheath (Fig. S3A) and the DTC (Fig. S3B) or any change in the number of sperm nuclei that the animals produce (Fig. S3C, D). These observations confirm that impaired differentiation of germ cells to oocytes leads to germline tumor development in rpom-1 (RNAi)-treated animals. 


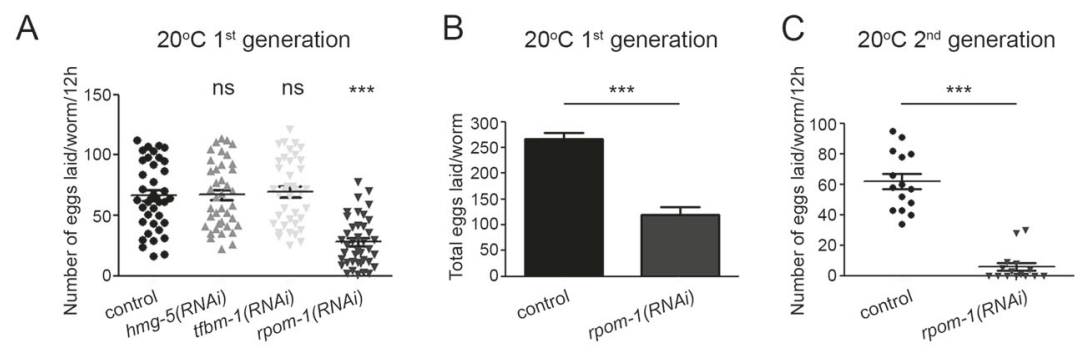

D
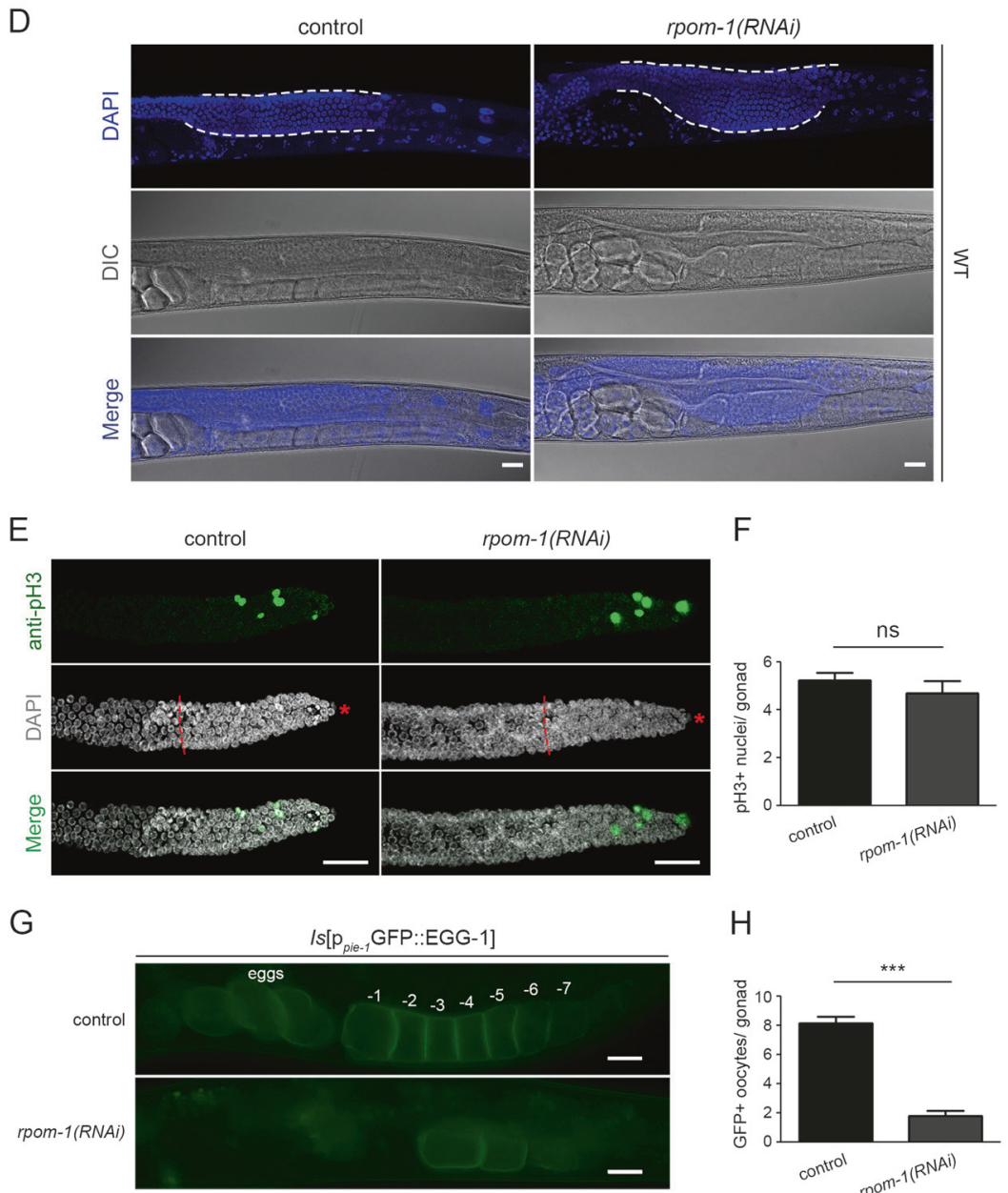

$\mathrm{H}$
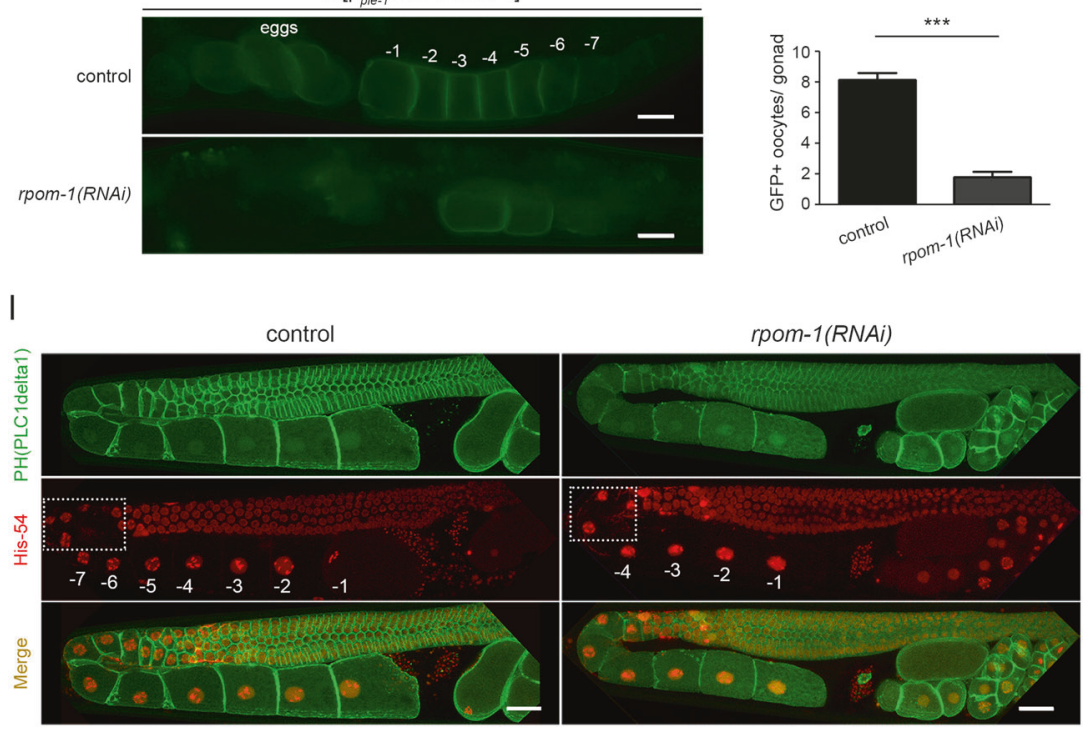
Fig. 1 RPOM-1 depletion causes germline tumor formation in C. elegans. a, b The brood size of rpom-1(RNAi)-treated hermaphrodites is significantly reduced (up to 50\%) compared to their control counterparts. Unpaired $t$-test was used for the estimation of statistical significance $(n>$ $40 ; * * * P<0.001)$. c Egg laying measurement of animals treated with control or rpom-1(RNAi) for two subsequent generations. d DAPI staining of day 1 control and rpom-1(RNAi)-treated WT animals. Inhibition of mitochondrial transcription results in germ nuclei arrest in the pachytene region of prophase I in the germline syncytium. The dashed lines surround the germline syncytium. e Phosphorylated histone H3 antibody staining of extruded gonads for the detection of mitotic nuclei in the distal gonad arm. Red dashed lines highlight the border between the mitotic region and the transition zone, marked by the appearance of crescentshape nuclei, while the red asterisk marks the relative position of the distal gonad arm tip. f Quantification of phosphorylated histone H3 positive germ nuclei in control and rpom-1(RNAi)-treated hermaphrodites. g Representative images of EGG-1 positive oocytes in the proximal arm from control and RPOM-1-depleted gonads. h Quantification of EGG-1 positive oocytes in control and rpom-1(RNAi)-treated hermaphrodites. i Confocal image of day 1 transgenic worms expressing a cell membrane tagged GFP and a histone-54 fused mCherry. Animals treated with rpom1(RNAi) display a lower number of nuclei in diplotene (compare dashed rectangles) and fewer mature oocytes in diakinesis. -1 denotes the most proximal oocyte. Unpaired $t$-test was used for the estimation of statistical significance $(n>40 ; * * * P<0.001)$. Error bars, s.e.m. Images were acquired using a $\times 40$ objective lens. Scale bars, $20 \mu \mathrm{m}$

\section{Germline apoptosis counterbalances tumor formation}

We surveyed for cellular responses triggered by aberrant mitochondrial biogenesis. RPOM-1 depletion resulted in accumulation of apoptotic corpses in the gonad syncytium, which were clearly evident using differential interference contrast (DIC; Nomarski) microscopy and a CED-1::GFP reporter strain (Fig. 2a). CED-1 is a transmembrane receptor which normally clusters around apoptotic corpses before they are engulfed and removed by gonad sheath cells [26, 27]. Furthermore, both HMG-5 and TFBM-1 depletion efficiently induced apoptosis, although to a lesser extent than rpom-1(RNAi) (Fig. 2b), indicating that distinct signals emanating from dysfunctional mitochondria may trigger programmed cell death. Inhibition of apoptosis in homozygous ced-3/caspase mutants can lead to the accumulation of germ cell corpses that cannot be removed [28]. Notably, rpom-1 knockdown in ced-3(n717) homozygous mutants aggravated the pachytene arrest phenotype observed in wild-type nematodes. By contrast, loss of function mutants
A

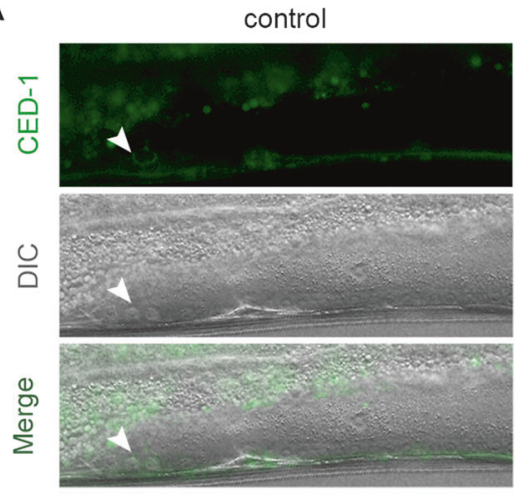

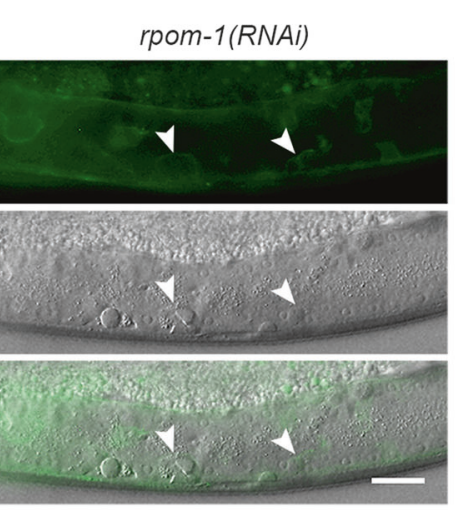

control
B

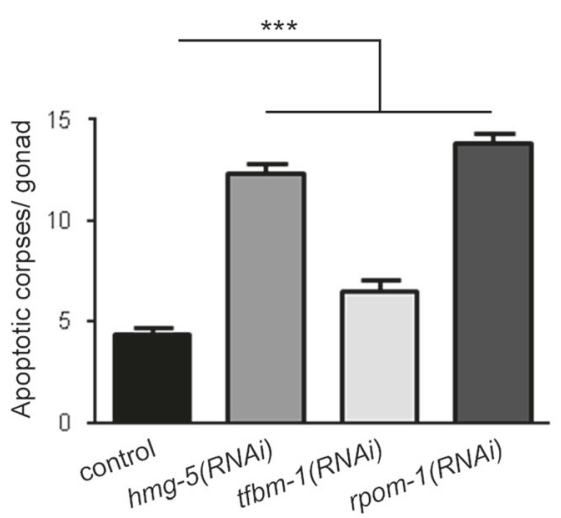

C
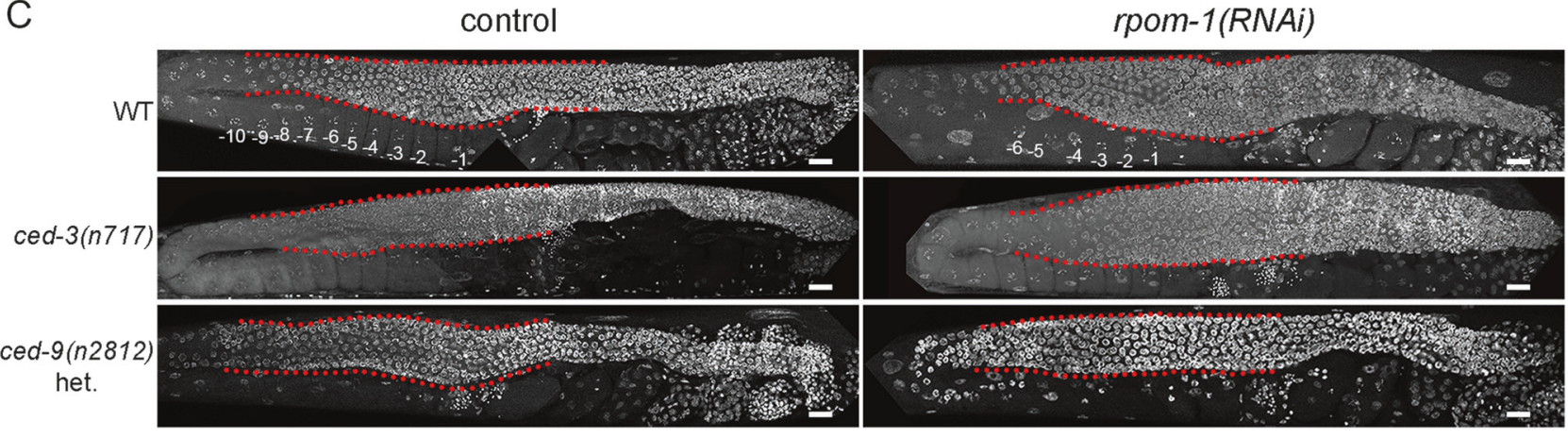

Fig. 2 Induction of apoptosis alleviates germline tumor development. a Apoptosis induction following rpom-1 downregulation, as monitored using the CED-1::GFP reporter in combination with DIC microscopy. Arrows highlight apoptotic corpses in the syncytium area. b A twofold induction in the number of early apoptotic corpses can be detected upon RPOM-1 depletion. Hmg-5 and tfbm-1 downregulation also trigger apoptosis $(n=40 ; * * * P<0.001$, one-way ANOVA was used for multiple comparisons). c RPOM-1 depletion in $\mathrm{ced}-3(n 717) / \mathrm{cas}$ pase-deficient animals causes pronounced tumor formation, even more severe than in wild-type worms. Heterozygous ced-9(n2812)/BCL-2 animals exhibit no sign of germ nuclei arrest in pachytene. The red dashed lines surround the pachytene region of the gonads. -1 denotes the most proximal oocyte. Images were acquired using a $\times 40$ objective lens. Error bars, s.e.m. Scale bars, $20 \mu \mathrm{m}$ 
for ced-9(n2814)/BCL-2, where apoptosis is induced, exhibit no signs of tumor formation upon rpom-1 downregulation (Fig. 2c). Hence, induction of apoptosis compensates for the tumor phenotype caused by the inhibition of mitochondrial transcription.

\section{Mitochondrial transcription acts in parallel with signaling pathways converging on the germline}

We next investigated the impact of RPOM-1 depletion in mutants with reported defects in germline homeostasis. Insulin/IGF-1 signaling promotes proliferation of germline stem cells [29]. While rpom-1 downregulation caused tumor formation in the germline syncytium of wild-type animals
(Fig. 3a, b), it differentially affected daf-2(e1370)/IGFR mutants by producing dwarf gonads at permissive temperatures $\left(20^{\circ} \mathrm{C}\right)$ and aggravating their proliferative defects (Fig. 3c, d). TGF- $\beta$ signaling is also reported to affect the balance between mitosis and differentiation in the $C$. elegans germline, in response to environmental cues, such as concentration of dauer pheromone or population density [30]. RPOM-1 depletion in daf-1(m40)/TGFR mutants behaved similarly to $d a f-2(e 1370) / \mathrm{IGFR}$ mutants, since it produced gonads with further compromised proliferative potential compared to their respective controls (Fig. 3e, f). In sharp contrast the gonads of rsks-1(ok1255)/S6K mutants, which exhibit attenuated global protein synthesis, were identical to those of control animals (Fig. 3g, h). Importantly, ife-5

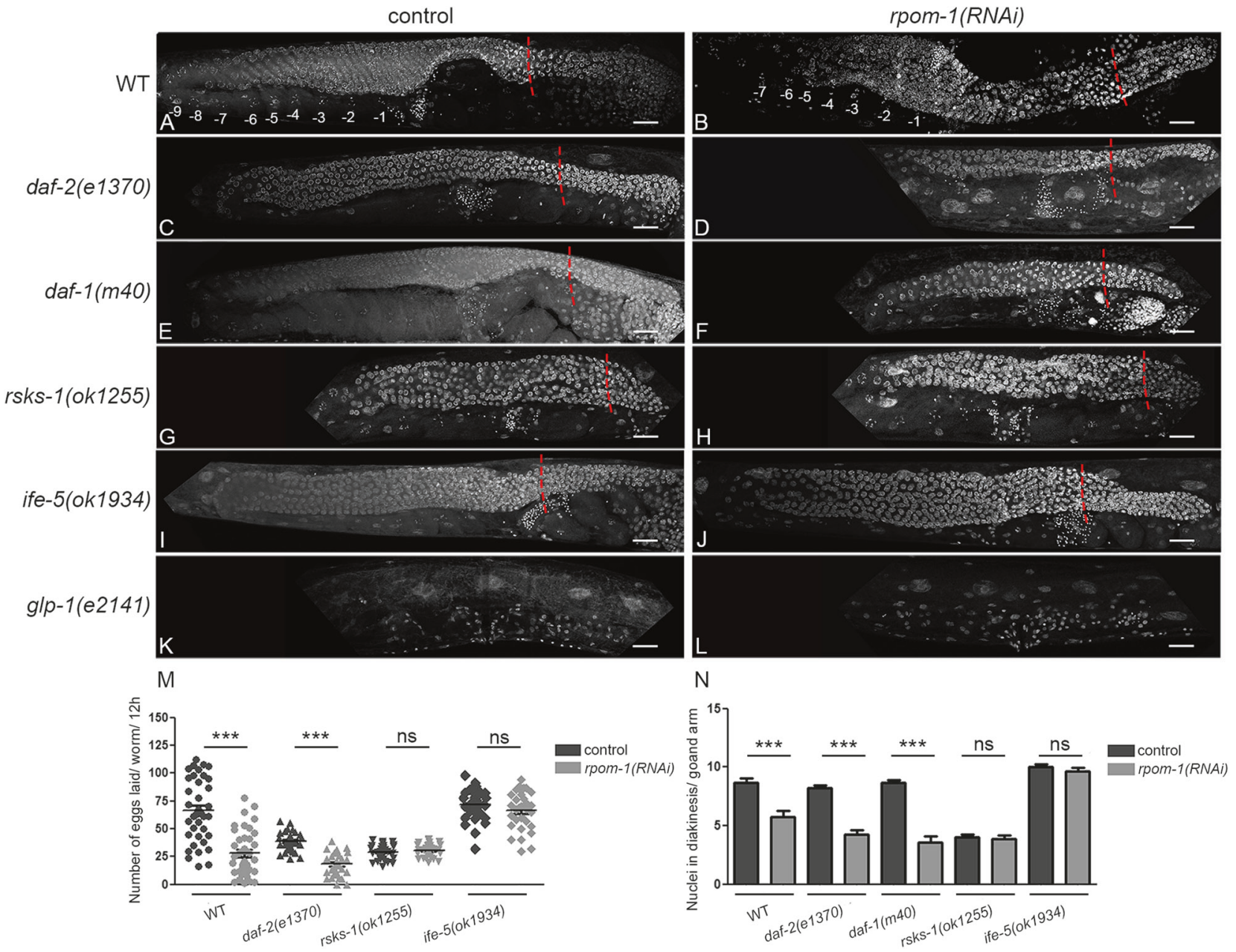

Fig. 3 Mitochondrial transcription acts in parallel with signaling pathways converging on the germline. Hoechst 33342 staining of D1 adult animals in control conditions and upon rpom-1 silencing. a, b Inhibition of mitochondrial transcription causes pachytene arrest in otherwise wild-type animals. In contrast, treatment of daf-2/ IGFR (c, d) and daf-1/ TGFR (e, f) homozygous mutants with rpom-1(RNAi) at $20^{\circ} \mathrm{C}$ produces dwarf gonads and augments their reported defects. Mutants with attenuated protein synthesis rates, such as rsks-1/ S6K $(\mathbf{g}, \mathbf{h})$ and ife-5/eIF4E $(\mathbf{i}, \mathbf{j})$ are indistinguishable from their control counterparts upon treatment with rpom-1(RNAi). k, l Glp-1/ Notch loss of function produces germline-less animals at restrictive temperatures. The red dashed lines indicate the border between the mitotic region and the transition zone. $\mathbf{m}$ Egg laying measurements in wild-type, daf2(e1370), rsks-1(ok1255) and ife-5(ok1934) mutants. n Quantification of the number of germ nuclei reaching diakinesis in wild-type, daf-2 (e1370), daf-1(m40), rsks-1(ok1255) and ife-5(ok1934) genetic backgrounds. $(n>40 ; * * * P<0.001$, unpaired $t$-test). Error bars, s.e.m. Images were acquired using a $\times 40$ objective lens. Scale bars $20 \mu \mathrm{m}$ 
(ok1934)/eIF4E mutants, similarly to rsks-1(ok1255) mutant animals, remained unaffected by RPOM-1 depletion (Fig. 3i, j). Furthermore, the egg-laying defect caused by RPOM-1 deficiency was completely absent in protein synthesisdefective nematodes, as evident by counting the brood size of individual animals and the number of diakinesis-staged nuclei (Fig. 3m, n). This indicates that reduced protein synthesis provides a selective advantage under conditions of impaired mitochondrial ATP production. Finally, rpom-1 (RNAi)-treated $g l p-1(e 2141) /$ Notch loss of function mutants was indistinguishable from controls under restrictive temperatures, producing germline-less hermaphrodites (Fig. 3k, 1). Numerous mutant strains for vital germline components, such as P-granules, are fully fertile at standard growth conditions $\left(20^{\circ} \mathrm{C}\right)$, but become sterile following a switch to a higher temperature $\left(25^{\circ} \mathrm{C}\right)$ [31]. The defects accompanying RPOM-1 depletion became more prominent when the animals were raised at $25^{\circ} \mathrm{C}$. The gonads at that temperature virtually collapse, barely produce a few diplotene and diakinesis-staged germ nuclei and accumulate dead corpses (Fig. S4). Together, these findings suggest that the precise coordination of signaling pathways (Insulin/IGF-1, TGF- $\beta$, and Notch/Delta) and internal gonad processes (mitochondrial transcription and protein synthesis) shapes the balance between mitosis and meiosis in the C. elegans germline.

\section{rpom-1 expression is compartmentalized}

To monitor rpom-1 expression in vivo, we generated a translational reporter by fusing GFP to the carboxyl terminus of full-length rpom- 1 cDNA regulated by its endogenous operon promoter. rpom- 1 is expressed in several somatic tissues, including muscles, intestine, vulva, and neuronal cells in the nerve ring and the tail, in a pattern reminiscent of proteins localizing in the mitochondrial matrix (Fig. S5). rpom-1 is also strongly expressed in the germline of hermaphrodite animals in a punctuate pattern. In the gonads, mitochondria surround and enwrap germ cell nuclei in the syncytium region (Fig. S6A, B). We observed a clear colocalization of RPOM-1 with TMRE, a potentialdependent mitochondrial dye (Fig. S6C). We crossed RPOM-1::GFP transgenic animals with $\mathrm{p}_{\text {lag-2 }} \mathrm{MYR}:$ :tdTomato reporter animals, to visualize RPOM-1 expression in regard to the position of the DTC. We noticed that rpom-1 expression is low in the mitotic region distally, but increases profoundly as germ cell nuclei mature to form oocytes (Fig. 4a, Fig. S7A). Interestingly, RPOM-1 expression increases at the onset of pachytene, the exact same region where germ nuclei arrest upon RPOM-1 depletion. To achieve a faithful reconstitution of the endogenous rpom-1 expression pattern, we also generated a reporter strain that expresses a transgene carrying the endogenous rpom-1 $3^{\prime}$

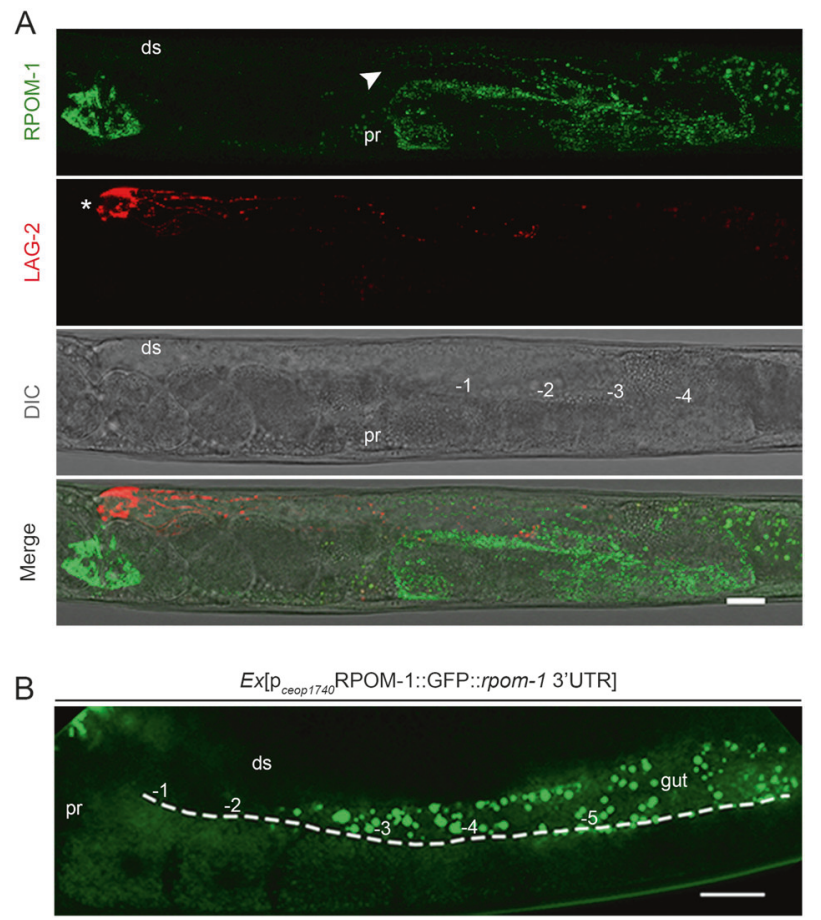

Fig. 4 Rpom- 1 expression is compartmentalized. a RPOM-1 abundance (in green) is low in the distal arm and the mitotic region of the gonads, becomes evident at the onset of the pachytene region (arrowhead) and substantially increases close to the turn and in the proximal arm, where the oocytes mature. LAG-2::myr::tdTomato (in red) marks the distal tip cell (white star) and its membrane projections. b A reporter strain overexpressing RPOM-1 under the control of its endogenous promoter and 3'UTR reveals higher expression in the oocytes and lower in the syncytium. Ds; distal, pr; proximal, -1 denotes the most proximal oocyte. Images were acquired using $\times 40$ and $\times 63$ objective lenses. Scale bars, $20 \mu \mathrm{m}$

UTR. On a similar note, RPOM-1 was enriched in the oocytes of the proximal gonad arm (Fig. 4b). The expression of the operon's promoter per se gradually increases in a distal to proximal manner (Fig. S7B). IFET-1, which localizes to P-granules and is required for normal gonad development, is a general translational repressor in the germline [32]. Treatment of our reporter animals with ifet-1 (RNAi) de-repressed rpom-1 expression distally (Fig. S7C). Hence, rpom-1 expression in the germline is compartmentalized, increases as germ nuclei progress to the proximal arm and form oocytes and is directly regulated by IFET-1 at the translational level.

\section{Transition to tubular mitochondria is a hallmark of differentiation}

We noticed that mitochondrial morphology alters in the course of germ cell differentiation. The distal gonad arm was abundant with globular mitochondria, while elongated organelles prevailed in the proximal arm (Fig. 5a, e). In the gonad turn both globular and tubular mitochondria coexist 

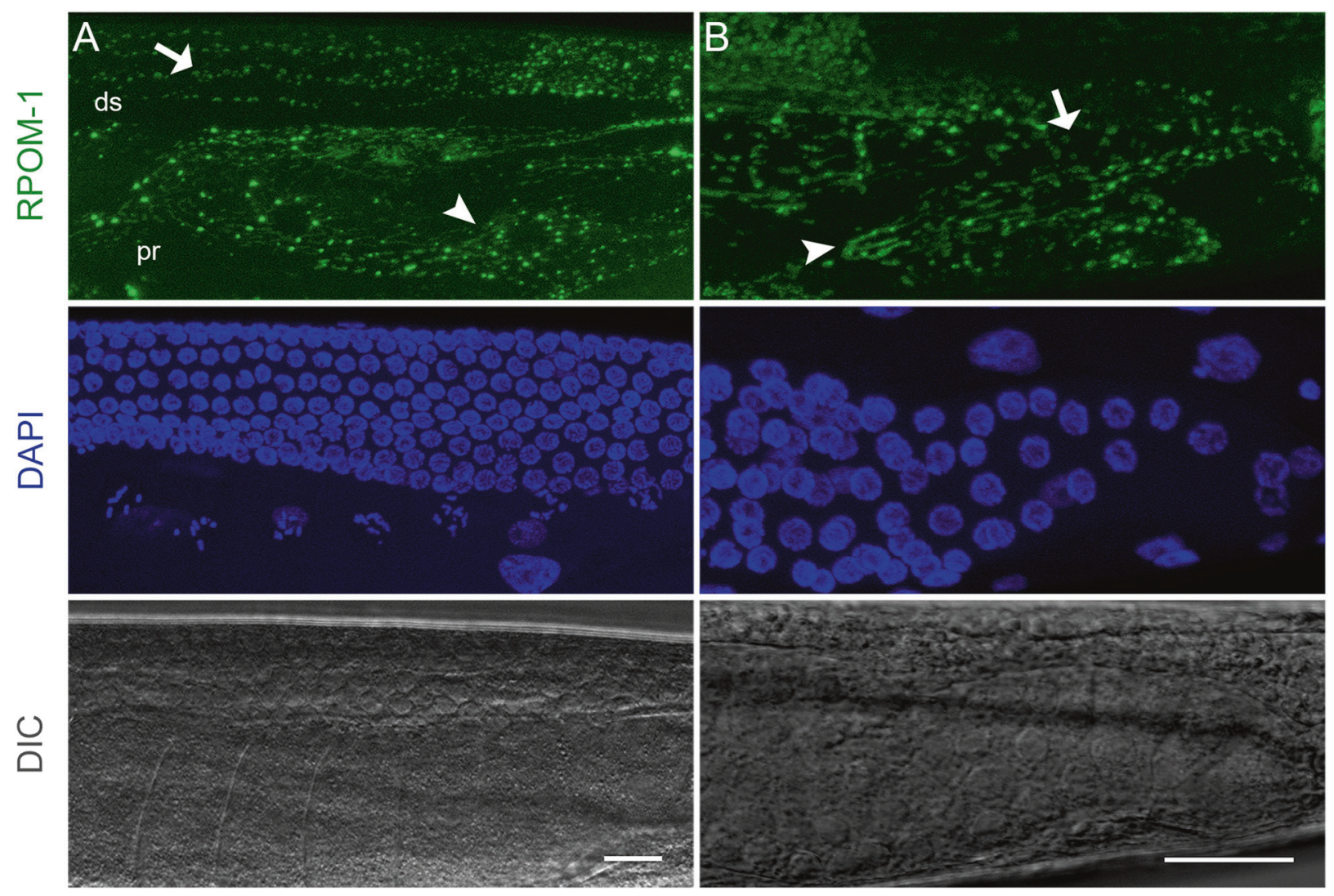

C

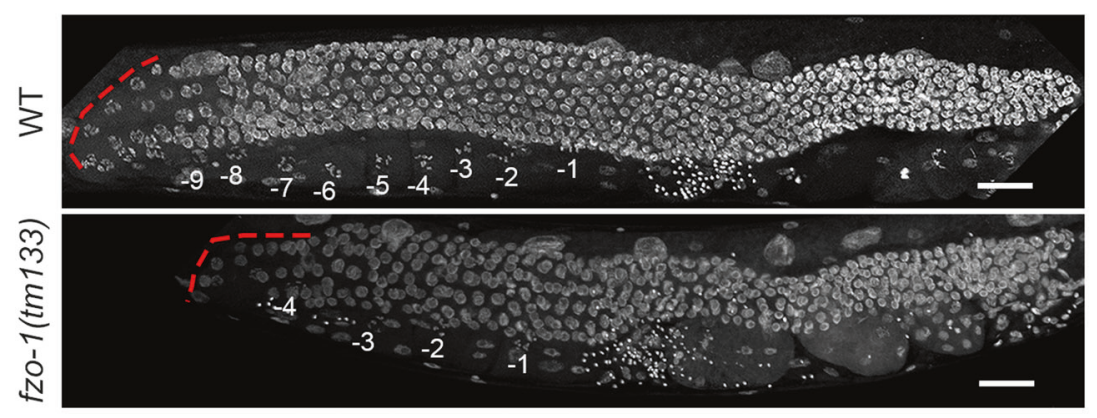

$\mathrm{E}$

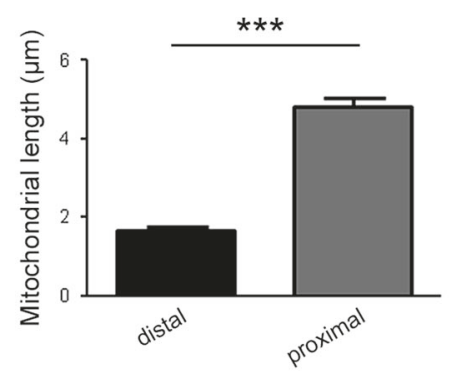

D

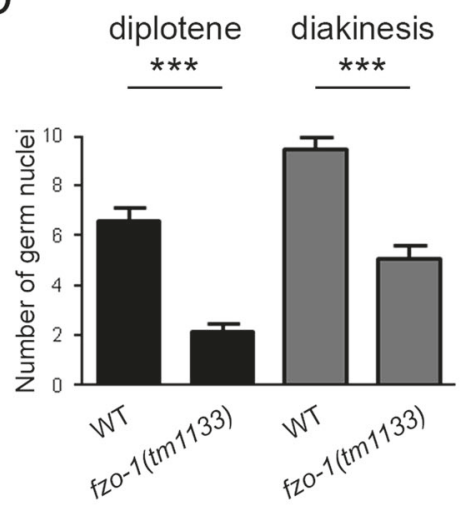

$\mathrm{F}$

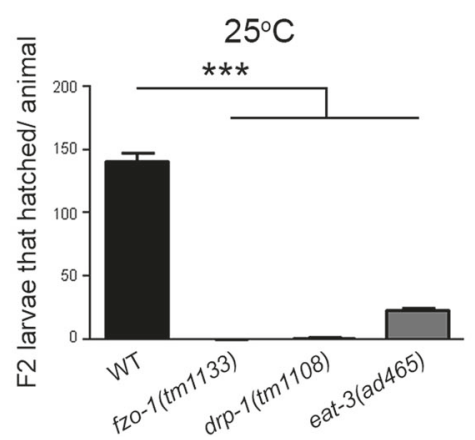

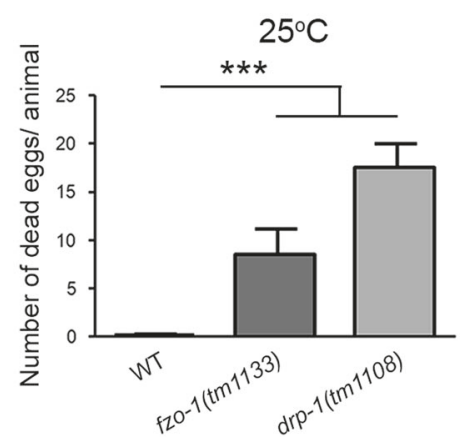

(Fig. 5b). In that region, an actin-dependent cytoplasmic streaming deposits cytoplasmic material and mitochondria to the oocytes [33]. Accumulating evidence suggests that elongated mitochondria are associated with elevated ETC activity and vice versa [34, 35]. Mitochondrial shape is malleable and alters to fulfill physiological demands, in 
Fig. 5 Transition from globular to tubular mitochondria is a prerequisite for germline homeostasis. a Confocal image of an adult $C$. elegans gonad. In the distal arm, mitochondria have a globular shape (arrow), which gradually switches to a more elongated/ tubular one in the oocytes of the proximal arm (arrowhead). b The turn of the gonad, shown in magnification, is the site where the shape alteration occurs. There, both globular (arrow) and tubular (arrowhead) mitochondria can be observed. c DAPI staining of wild-type and fzo-1(tm1133)/ Mitofusin homozygous mutants. The red dashed line marks the gonad turn. d Day 1 adult fzo-1(tm1133)/Mitofusin mutant animals, bearing defects in mitochondrial fusion, produce significantly fewer germ nuclei in diplotene as well as nuclei in diakinesis compared to their control counterparts. ( $n>40$; $* * * P<0.001$, unpaired $t$-test). e Quantification of mitochondrial length in the proximal and distal arm of the gonads. f Animals with perturbed mitochondrial dynamics (fusionfission), such as $f z o-1$, drp- 1 and eat- 3 mutants become sterile when exposed to a mild heat stress $\left(25^{\circ} \mathrm{C}\right)$. One-way ANOVA was used for the estimation of statistical significance $(n>40 ; * * * P<0.001)$. Error bars, s.e.m. Images were acquired using X40 and X63 objective lenses. Ds; distal, pr; proximal, -1 denotes the proximal-most oocyte. Scale bars, $20 \mu \mathrm{m}$

response to stress and other intracellular or environmental signals. Specialized dynamin GTPases FZO-1/Mitofusin and EAT-3/OPA-1 mediate fusion, while DRP-1 is required for fission [36]. Interestingly, fzo-1(tm1133) mutant animals possess significantly less germ nuclei in diplotene as well as oocytes in diakinesis, producing fewer offspring (Fig. 5c, d). Furthermore, while fzo-1(tm1133) and drp-1(tm1108) mutants were viable and fertile at $20^{\circ} \mathrm{C}$, they became sterile when the rearing temperature was shifted to $25^{\circ} \mathrm{C}$, producing mainly dead eggs and negligible offspring (Fig. 5f). Together, these findings support the notion that tubular mitochondria in the proximal arm represent the outcome of a maturation process, which is essential for oocytes to cope with their high-energy demands.

To shed light on the molecular mechanism that governs the alteration of mitochondrial morphology, we focused on well characterized pathways associated with germ cell differentiation in C. elegans. Spatial activation of MPK-1/ MAPK signaling is crucial for germ cell exit from pachytene. MPK-1 deficient mutants exhibit pachytene arrest, phenocopying RPOM-1 depletion [37]. Interestingly, tubular organelles were absent upon knockdown of mek-2 or $m p k-1$, the homologs of mammalian MEK and ERK kinases, respectively (Fig. 6a, c, f). GLD-1 is an RNA-binding protein that binds to the $3^{\prime} \mathrm{UTR}$ of target mRNAs, repressing their translation. In gld- 1 loss of function mutants germ nuclei exit meiosis and return to mitosis, forming germline tumors [38]. Similarly to FZO-1/Mitofusin depleted animals, gld-1(RNAi)-treated animals contain exclusively globular mitochondria in the proximal gonad arm (Fig. 6a, b, f). Gonad sheath cells are known to respond to spermderived signals (major sperm proteins) and promote oocyte maturation through activation of the $\mathrm{G} \alpha_{\mathrm{s}}$-adenylate cyclaseprotein kinase A pathway [39]. Inhibition of gsa-1, the worm Gs alpha subunit of heterotrimeric $G$ proteins, prevents oocyte maturation [40]. Interestingly, mitochondria failed to elongate in the proximal arm of $g s a-1(R N A i)$ treated animals (Fig. 6a, e, f) and the existing organelles were not efficiently polarized (Fig. 6g, h). Conversely, inhibition of GOA-1, a negative regulator of oocyte maturation, boosted mitochondrial potential in the proximal arm (Fig. 6g, h). Altogether, these findings suggest that germ nuclei differentiation is intertwined with mitochondrial maturation and the latter appears to rely on two important signaling pathways, namely MPK-1/MAPK and MSP.

\section{Mitochondria functionally mature en route to germ cell differentiation}

The established interplay between mitochondrial morphology and metabolic activity [34] prompted us to test mitochondrial maturation within the germline. We cloned Perceval, a fluorescent sensor for adenylate nucleotides [41], downstream of the pie-1 promoter, to uniformly express it in the germline. We also utilized the $t b b-23^{\prime} \mathrm{UTR}$ to avoid undesired silencing of our transgene [42]. Interestingly, we detected expression in the oocytes, but not in the gonad syncytium (Fig. 7a). This is consistent with the notion that ATP is produced proximally, in the area that is abundant with tubular mitochondria. Treatment with Antimycin A, a bacterial toxin that inhibits ETC complex III, dampened fluorescence in oocytes and fertilized eggs, proving that Perceval can indeed detect changes in mitochondrial ATP production (Fig. S8). To further verify the previous finding, we stained whole animals with dyes that stain mitochondria in a membrane potential-dependent manner, such as TMRE, DIOC6(3) (3,3'-Dihexyloxacarbocyanine Iodide), and MitoTracker Red CM-H2XROS. In congruence with Perceval findings, mitochondrial membrane potential and ROS levels were elevated in developing oocytes (Fig. 7b-d). Interestingly, rpom-1 knockdown inhibited mitochondrial polarization in the proximal gonad arm as indicated by DIOC6 (3) staining (Fig. S9). We postulate that mitochondrial elongation is a primary step in a maturation process, which results in enhanced mitochondrial polarization and concomitant ATP and ROS production in oocytes.

\section{Elevated POLRMT expression and switch to tubular mitochondria are conserved during evolution}

We next wondered whether a similar mechanism controls mouse stem cell differentiation. We employed J1 cells, derived from the inner cell mass of mouse blastocysts and grown on spherical colonies in the presence of LIF cytokine. We removed LIF from our culture medium and let the cells differentiate in an unbiased manner. We stained with antibodies for POLRMT and MTCO1, the cytochrome c oxidase subunit I encoded by the mitochondrial genome. 

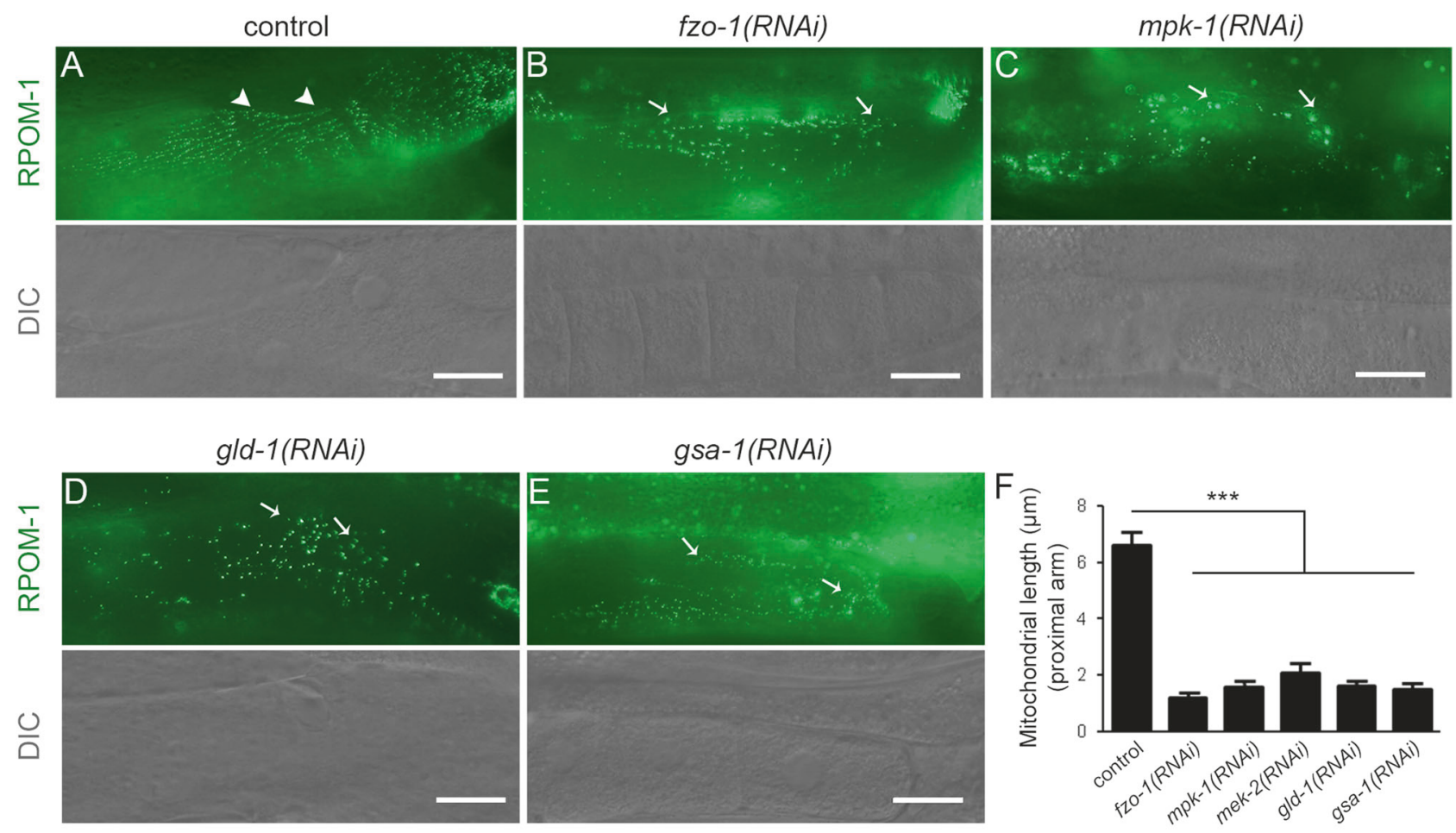

G

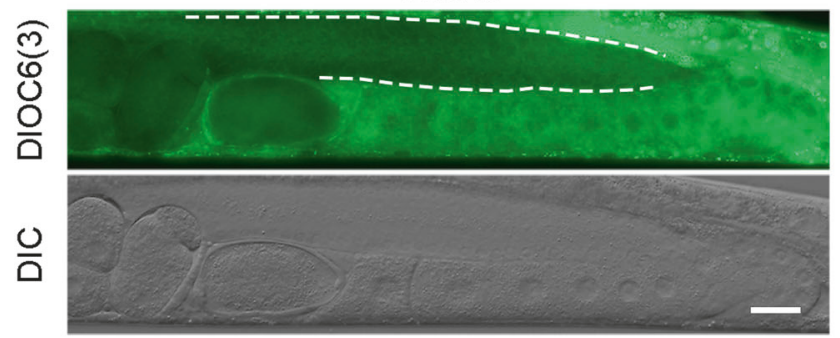

goa-1(RNAi)

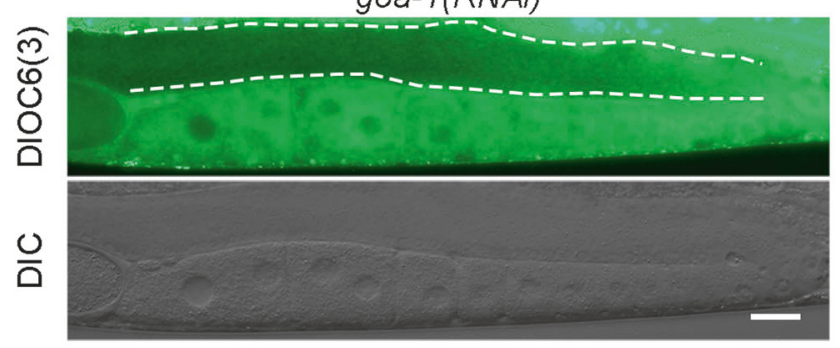

Fig. 6 Sperm-derived signals promote mitochondrial maturation. a Mitochondria in the proximal gonad arm are tubular under control conditions (arrowheads). b Knockdown of $f z o-1$ leads to mitochondrial network fragmentation and globular mitochondria in the proximal gonad arm (arrows). c Inhibition of MPK-1/MAPK signaling via $m p k$ 1(RNAi) results in a failure of mitochondria to elongate proximally (arrows). d Mitochondria are exclusively globular in the proximal arm of $g l d-1(R N A i)$-treated animals (arrows). e GSA-1 inhibition results in failure of oocyte maturation as well as mitochondrial elongation. f Quantification of mitochondrial length in proximal gonad arm oocytes upon the respective RNAi treatments $(n=40 ; * * * P<0.001$,

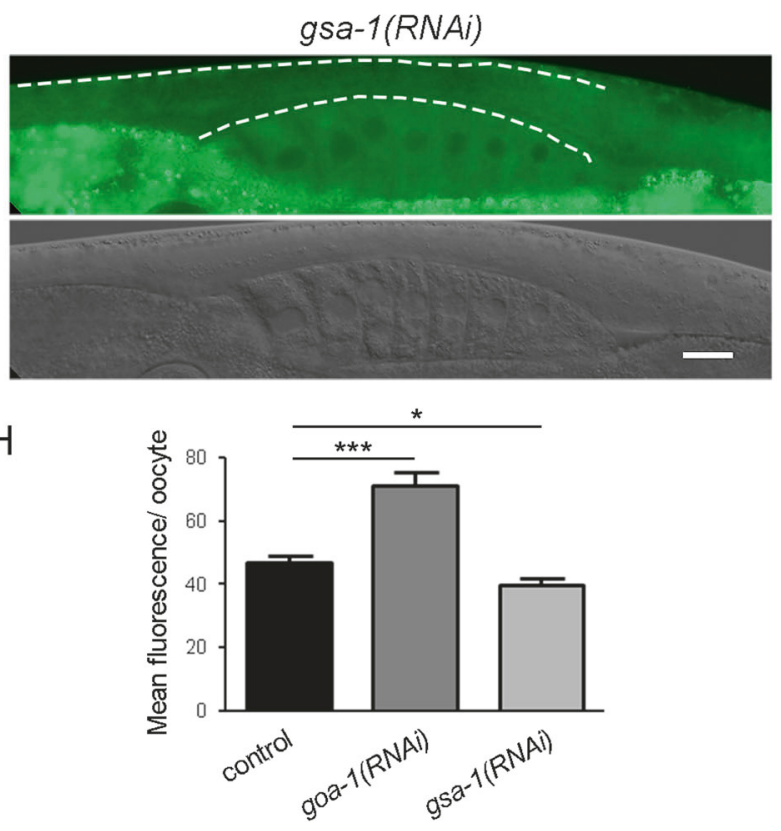

one-way ANOVA was used for multiple comparisons). g DIOC6(3) staining reveals that mitochondria polarize in the course of germ cell differentiation. Inhibition of GOA-1, a negative regulator of sperm signaling, boosts mitochondrial potential in the proximal gonad arm. Treatment with $g s a-1(R N A i)$ results in a failure of mitochondria to polarize proximally. h Quantification of the DIOC6(3) fluorescence per oocyte of the proximal gonad arm. The dashed lines surround the germline syncytium. $(n=40 ; * * * P<0.001$, unpaired $t$-test $)$. Error bars, s.e.m. Images were acquired using a X40 objective lens. Scale bar, $20 \mu \mathrm{m}$ 

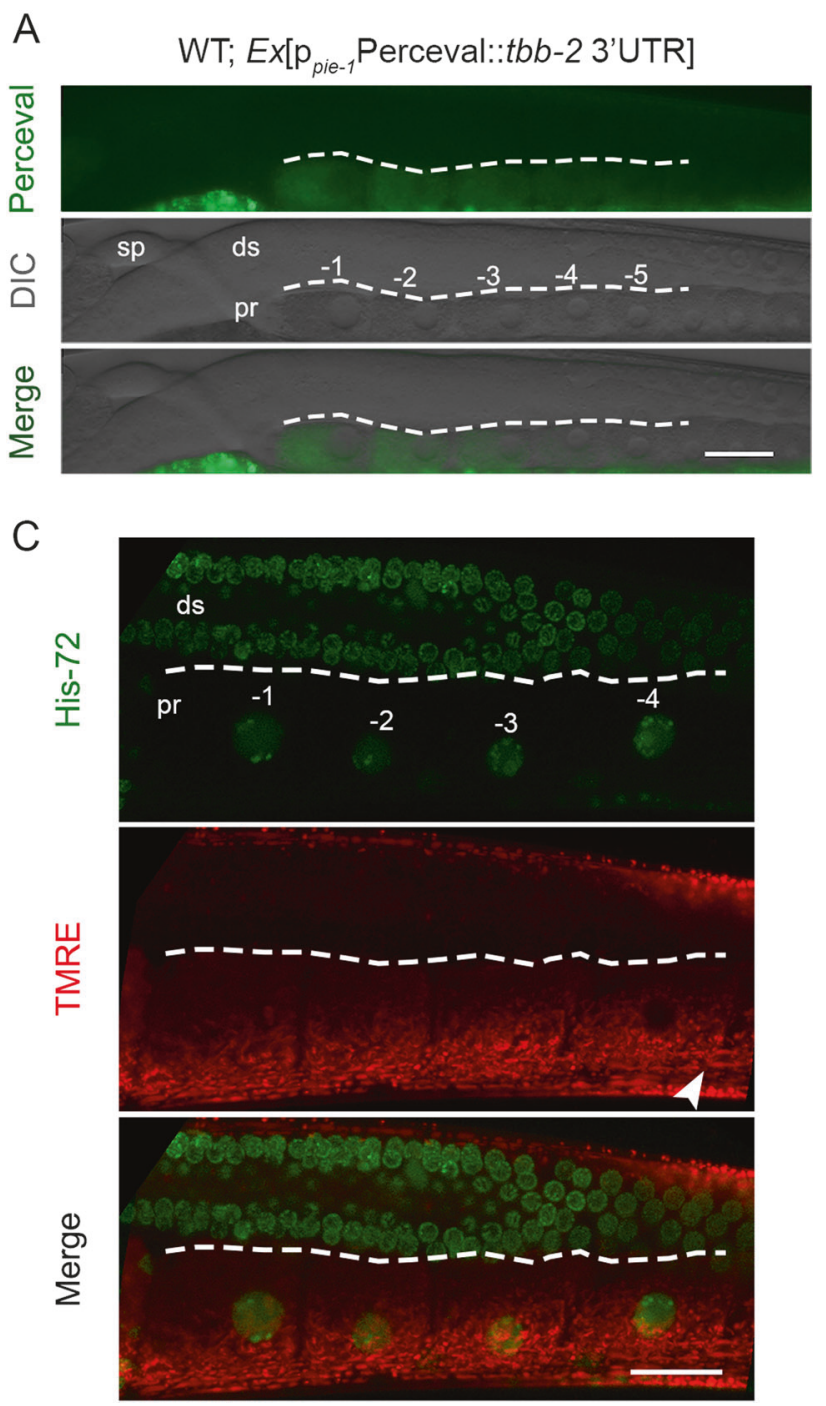

Fig. 7 Mitochondria functionally mature during germ nuclei differentiation. a The ATP/ADP sensor Perceval was overexpressed in the C. elegans germline under the control of pie-1 promoter, to achieve germline-specific expression. Perceval emission increases upon ATP binding. Fluorescence could be mainly detected in the oocytes, indicating increased ATP production in the proximal arm. b DIOC6(3) mitochondrial dye preferentially stains energized mitochondria in the

The staining was much weaker at the core of the stem cell colonies and progressively increased as cells differentiated and extended membrane projections typical of differentiated cells. Furthermore, $48 \mathrm{~h}$ after LIF removal, we observed POLRMT-positive tubular mitochondria (Fig. S10). We also simultaneously stained with POLRMT and OCT-4 antibodies. OCT-4 is a key pluripotency transcription factor that shuttles between the nucleus and the cytoplasm. Its nuclear retention enhances reprogramming efficiency and is associated with pluripotency [43]. Notably, OCT-4 retention in the nucleus was associated with reduced POLRMT expression, while cytoplasmic OCT-4 coincided with increased POLRMT expression (Fig. 8). Collectively, and
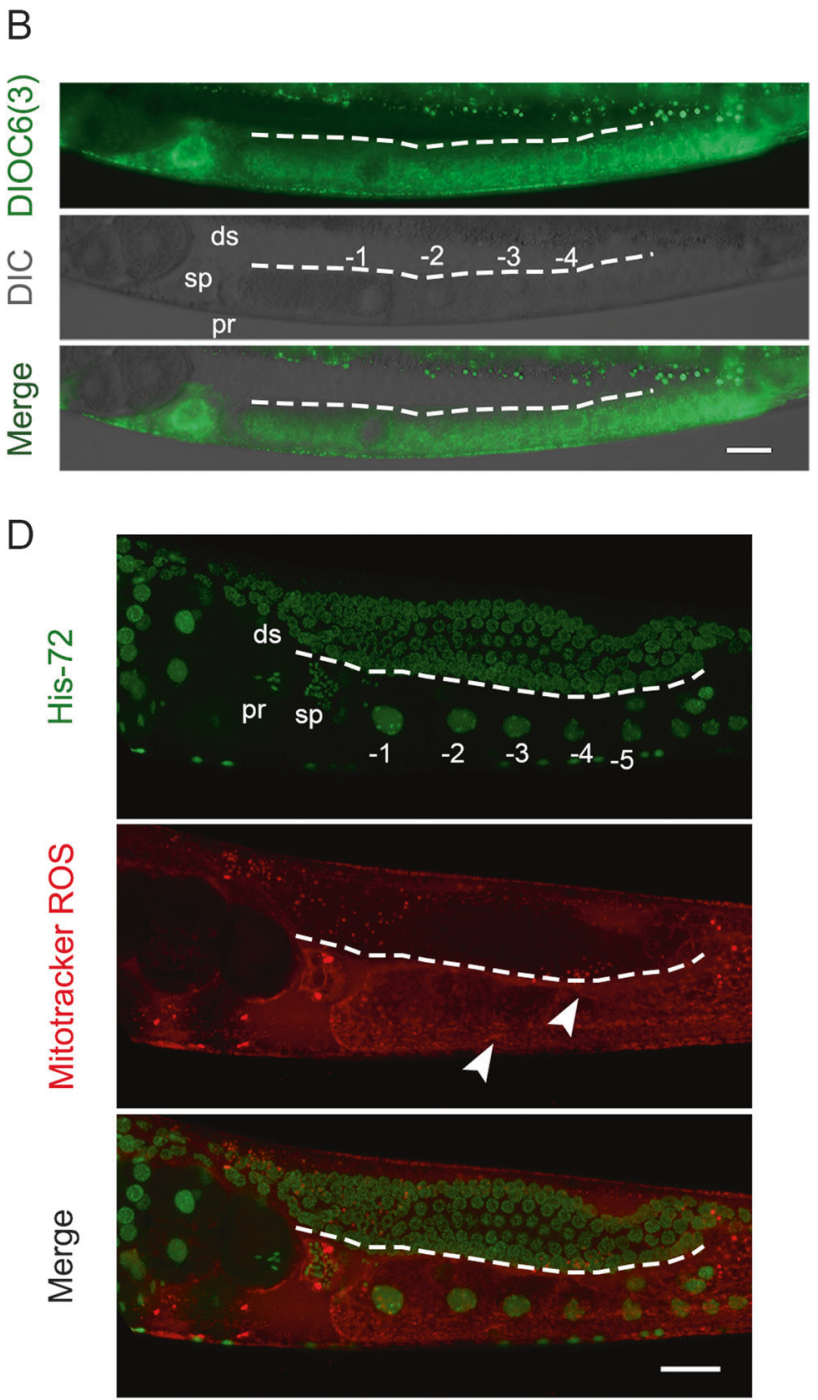

proximal gonad arm. c TMRE staining reveals increased electrochemical potential in the oocytes of the proximal arm. d Mitochondrial ROS production increases as the germ nuclei mature and give rise to oocytes. Arrowheads highlight tubular mitochondria in the proximal gonad arm. Sp; spermatheca, ds; distal, pr; proximal, -1 denotes the most proximal oocyte. Images were acquired using $\times 40$ and $\times 63$ objective lenses. Scale bars, $20 \mu \mathrm{m}$

in line with the $C$. elegans findings, mouse stem cells exhibit low POLRMT expression, while differentiation is accompanied by an increase in POLRMT expression and the appearance of elongated mitochondria.

\section{Discussion}

The term stem cell niche refers to the specific microenvironment, which ensures that stem cells are protected from harmful agents, divide and differentiate to constantly replenish organs [44]. Each C. elegans gonad hosts a unique stem cell niche in an otherwise postmitotic organism [45]. 


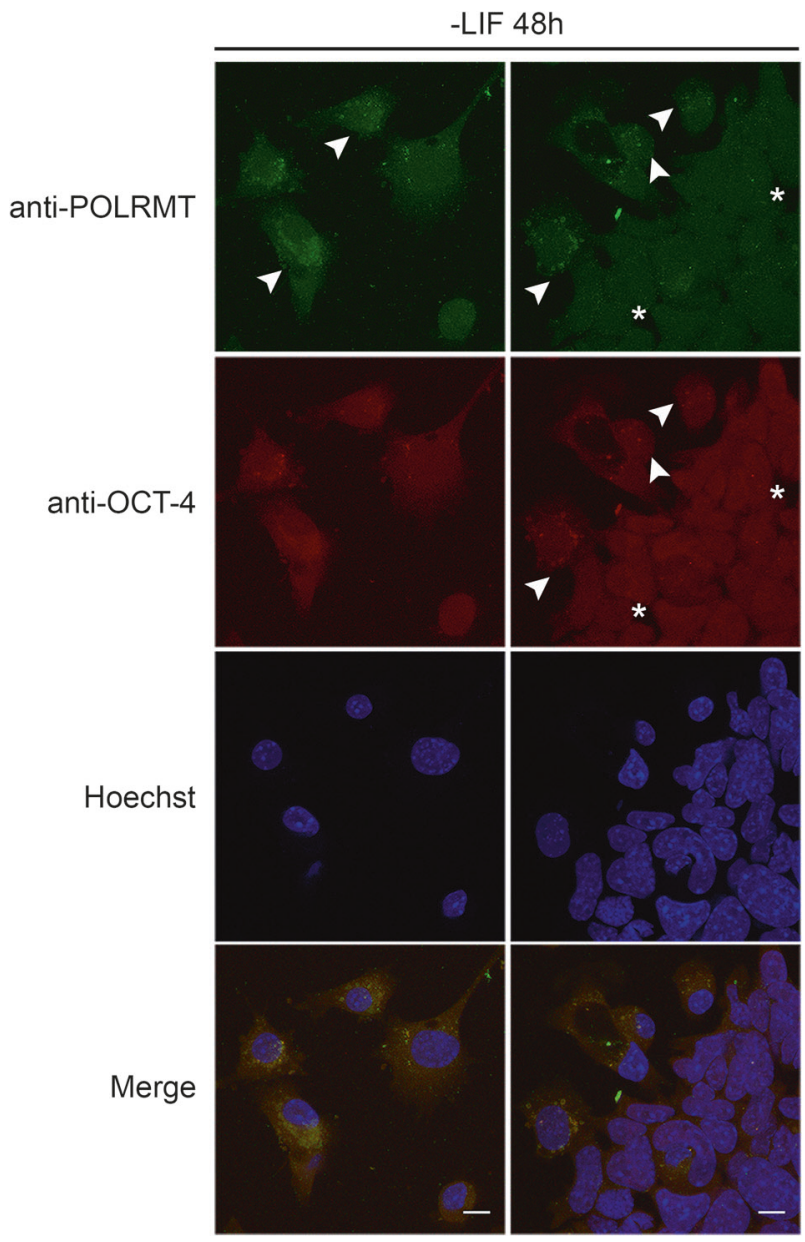

Fig. 8 Mammalian stem cell differentiation upon LIF removal is accompanied by an increase in POLRMT expression. Elevated POLRMT expression is observed in cells with increased cytoplasmic OCT-4 abundance (arrowheads). In contrast, adjacent areas with increased nuclear OCT-4 abundance (stars) display lower POLRMT expression. Images were acquired using a $\times 40$ objective lens. Scale bars, $20 \mu \mathrm{m}$

This is the main tissue where mitochondrial DNA replication occurs [46]. A previous study showed that mutation of a germline-specific mitochondrial ATPase subunit impairs fecundity [47]. We find that perturbation of mitochondrial biogenesis, energy production, and dynamics, collectively referred to as bioenergetics, can profoundly affect germ cell differentiation. We describe a maturation process, whereby globular, immature mitochondria are gradually converted to elongated, functional organelles to support increased oocyte energy demands (Fig. 9). This switch is tightly regulated by two core signaling pathways associated with oocyte production and maturation, namely MAPK/ERK and MSP. The MAPK/ERK pathway has pleiotropic functions in the $C$. elegans germline [37]. The DDX-19 helicase and GSK-3 kinase have been shown to be direct MPK-1 targets in vivo [48]. In addition, MPK-1 phosphorylates NOS-3, promoting degradation of TRA-1 by the FEM-CUL2 complex, to facilitate oocyte membrane organization [49]. MPK-1 phosphorylation itself is positively regulated by MSP signaling [50]. Thus, mitochondrial bioenergetics is likely a nodal point modulated by these cascades, in stem cells.

Our findings indicate that expression of mitochondrial RNA polymerase progressively increases during germ cell differentiation. This is accompanied by mitochondrial elongation and manifestation of several hallmarks of metabolic activity, such as increased electrochemical potential, ATP, and ROS production. Rpom-1 mRNA is one of the numerous targets of FBF-1, a Pumilio family, RNAbinding protein that negatively regulates the expression of mRNAs implicated in meiotic entry $[51,52]$. In addition, localized transcription and mRNA translation likely contribute to the subcellular compartmentalization of RPOM-1. Taken together, our observations indicate that upon perturbation of germ cell bioenergetics, germ nuclei stall in the pachytene stage and fail to differentiate, generating fewer oocytes. Consistent with this notion, mitochondrial ATP synthase function is required for the maturation of mitochondrial cristae in Drosophila ovaries [53]. Similarly, the pluripotent state of mammalian stem cells has been linked to decreased mitochondrial respiration, in favor of anaerobic glycolysis [54]. Previous studies have demonstrated that mitochondrial mass, mtDNA copy number, and oxygen consumption increase during stem cell differentiation [5557]. By contrast, successful induction of pluripotent stem cell (iPSC) lines is marked by a reduction of ETC function [58]. Hence, a switch to enhanced mitochondrial respiration is a prerequisite for stem cell differentiation across species.

Conserved signaling pathways implicated in lifespan regulation and dauer formation, also influence germline homeostasis. For instance, Insulin/IGF-1 promotes germ cell proliferation, while DAF-16/FOXO is beneficial for stem cell pool maintenance during ageing [29, 59]. Furthermore, TORC1 and RSKS-1/S6K are required for efficient proliferation of germ cell progenitors [60]. In addition, ASI neuron-derived TGF- $\beta$ signals determine the balance between mitosis and differentiation in the C. elegans germline [30]. Attenuation of Insulin/IGF-1 and TGF- $\beta$ signaling, combined with perturbation of mitochondrial transcription generates an atrophic germline, and exacerbates proliferation defects, indicating that mitochondria act in concert with extrinsic growth stimuli to dictate mitosis versus differentiation decisions. Similarly, energy is diverted to stress resistance and maintenance mechanisms in mutants with reduced protein synthesis $[61,62]$. In this context, as the disposable soma concept postulates, damage repair takes precedence over protein synthesis for germline maintenance and reproduction.

The precise balance between mitosis and differentiation is of utmost importance for tissue and organismal homeostasis. Our work provides novel insights on how mitochondrial bioenergetics dictates cell fate decisions and 


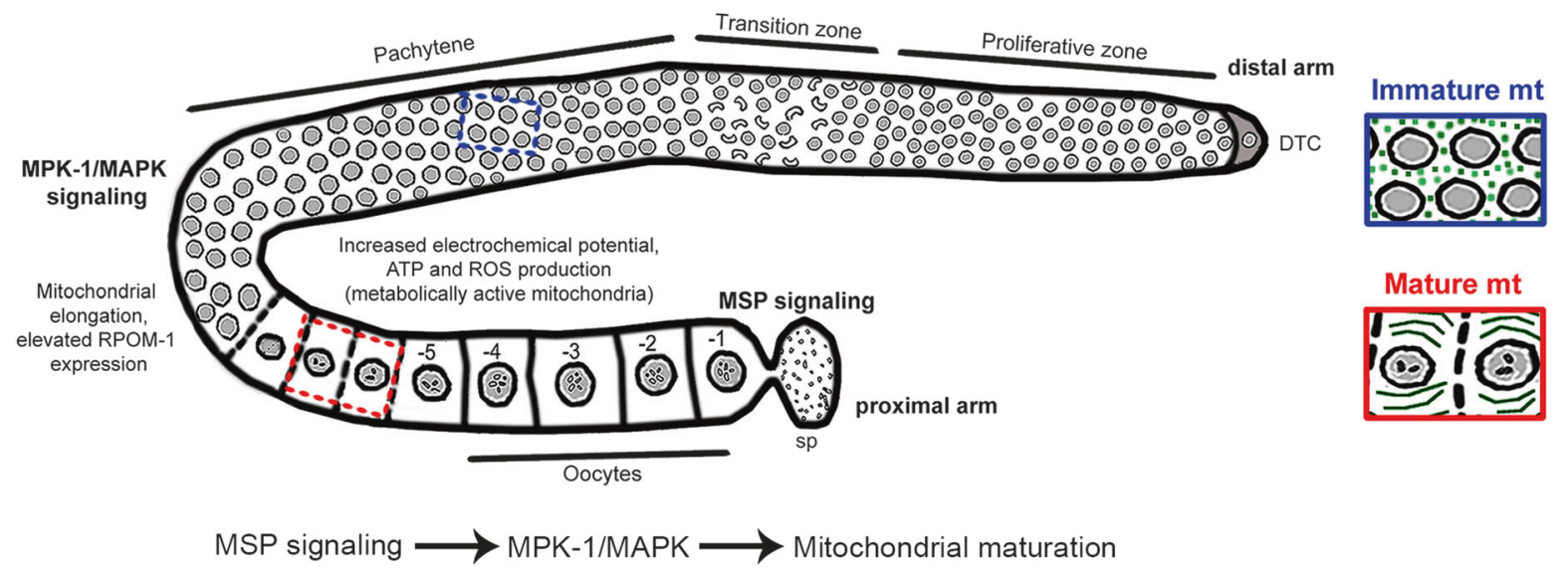

Fig. 9 Intact mitochondrial bioenergetics safeguards germline homeostasis. Expression of rpom-1 (the nematode orthologue of POLRMT) increases progressively as the germ nuclei mature and mitochondria acquire an elongated, tubular shape. The boost in mitochondrial

integrates mitochondria at the core of the developmental modules that shape the $C$. elegans germline. Perturbation of mitochondrial function obstructs germ nuclei differentiation and causes cancer-like phenotypes. A main challenge for future research is to delineate the molecular underpinnings of the germline mitochondrial metabolic switch as well as its temporal and spatial regulation.

Acknowledgements We thank A. Pasparaki and G. Tsikala for excellent technical support. We acknowledge E. Kyriakakis, K. Palikaras, and M. Markaki for critically reading the manuscript. We thank G. Yellen and Addgene for providing the pRsetB-his7-Perceval plasmid. Nematode strains used in this work were provided by the Caenorhabditis Genetics Center, which is funded by the National Centre for Research Resources of the National Institutes of Health, and S. Mitani (National Bioresource Project) in Japan. We thank A. Fire for plasmid vectors. This work was supported by the European Research Council (GA695190- MANNA), and the General Secretariat for Research and Technology of the Greek Ministry of Education (THALIS MIS380228 GEnAge).

\section{Compliance with ethical standards}

Conflict of interest The authors declare that they have no conflict of interest.

Publisher's note: Springer Nature remains neutral with regard to jurisdictional claims in published maps and institutional affiliations.

Open Access This article is licensed under a Creative Commons Attribution 4.0 International License, which permits use, sharing, adaptation, distribution and reproduction in any medium or format, as long as you give appropriate credit to the original author(s) and the source, provide a link to the Creative Commons license, and indicate if changes were made. The images or other third party material in this article are included in the article's Creative Commons license, unless indicated otherwise in a credit line to the material. If material is not included in the article's Creative Commons license and your intended use is not permitted by statutory regulation or exceeds the permitted use, you will need to obtain permission directly from the copyright metabolic activity is manifested by enhanced ATP and ROS production, as well as increased electrochemical potential in the proximal gonad arm. Mitochondrial maturation is under the control of MPK-1/ MAPK and MSP signaling pathways

holder. To view a copy of this license, visit http://creativecommons. org/licenses/by/4.0/.

\section{References}

1. Byrd DT, Knobel K, Affeldt K, Crittenden SL, Kimble JA. DTC niche plexus surrounds the germline stem cell pool in Caenorhabditis elegans. PLoS ONE. 2014;9:e88372.

2. Fox PM, Schedl T. Analysis of germline stem cell differentiation following loss of GLP-1 notch activity in Caenorhabditis elegans. Genetics. 2015;201:167-84.

3. Crittenden SL, Leonhard KA, Byrd DT, Kimble J. Cellular analyses of the mitotic region in the Caenorhabditis elegans adult germ line. Mol Biol Cell. 2006;17:3051-61.

4. Hirsh D, Oppenheim D, Klass M. Development of the reproductive system of Caenorhabditis elegans. Dev Biol. 1976;49:200-19.

5. Albert Hubbard EJ. Caenorhabditis elegans germ line: a model for stem cell biology. Dev Dyn. 2007;236:3343-57.

6. Folmes $\mathrm{CD}$, Ma H, Mitalipov S, Terzic A. Mitochondria in pluripotent stem cells: stemness regulators and disease targets. Curr Opin Genet Dev. 2016;38:1-7.

7. Archibald John M. Endosymbiosis and eukaryotic cell evolution. Curr Biol. 2015;25:R911-R21.

8. Tuppen HAL, Blakely EL, Turnbull DM, Taylor RW. Mitochondrial DNA mutations and human disease. Biochim et Biophys Acta. 2010;1797:113-28.

9. Schon EA, DiMauro S, Hirano M. Human mitochondrial DNA: roles of inherited and somatic mutations. Nat Rev Genet. 2012;13:878-90.

10. Amunts A, Brown A, Toots J, Scheres SHW, Ramakrishnan V. The structure of the human mitochondrial ribosome. Science. 2015;348:95-8.

11. Ringel R, Sologub M, Morozov YI, Litonin D, Cramer P, Temiakov D. Structure of human mitochondrial RNA polymerase. Nature. 2011;478:269-73.

12. Litonin D, Sologub M, Shi Y, Savkina M, Anikin M, Falkenberg M, et al. Human mitochondrial transcription revisited: only TFAM And TFB2M are required for transcription of the mitochondrial genes in vitro. J Biol Chem. 2010;285:18129-33.

13. Shi Y, Dierckx A, Wanrooij PH, Wanrooij S, Larsson N-G, Wilhelmsson LM, et al. Mammalian transcription factor A is a 
core component of the mitochondrial transcription machinery. Proc Natl Acad Sci USA. 2012;109:16510-5.

14. Sologub M, Litonin D, Anikin M, Mustaev A, Temiakov D. TFB2 is a transient component of the catalytic site of the human mitochondrial RNA polymerase. Cell. 2009;139:934-44.

15. Berman JR, Kenyon C. Germ-cell loss extends C. elegans life span through regulation of DAF-16 by kri-1 and lipophilichormone signaling. Cell. 2006;124:1055-68.

16. Kelly WG, Xu S, Montgomery MK, Fire A. Distinct requirements for somatic and germline expression of a generally expressed Caernorhabditis elegans gene. Genetics. 1997; 146:227-38.

17. Cristina D, Cary M, Lunceford A, Clarke C, Kenyon C. A regulated response to impaired respiration slows behavioral rates and increases lifespan in Caenorhabditis elegans. PLoS Genet. 2009;5:e1000450.

18. Palikaras K, Lionaki E, Tavernarakis N. Coordination of mitophagy and mitochondrial biogenesis during ageing in C. elegans. Nature. 2015;521:525-8.

19. Lui DY, Colaiacovo MP. Meiotic development in Caenorhabditis elegans. Adv Exp Med Biol. 2013;757:133-70.

20. Okimoto R, Macfarlane JL, Clary DO, Wolstenholme DR. The mitochondrial genomes of two nematodes, Caenorhabditis elegans and Ascaris suum. Genetics. 1992;130:471-98.

21. Sumitani M, Kasashima K, Matsugi J, Endo H. Biochemical properties of Caenorhabditis elegans HMG-5, a regulator of mitochondrial DNA. J Biochem. 2011;149:581-9.

22. Kukat C, Larsson N-G. mtDNA makes a U-turn for the mitochondrial nucleoid. Trends Cell Biol. 2013;23:457-63.

23. Cotney J, McKay SE, Shadel GS. Elucidation of separate, but collaborative functions of the rRNA methyltransferase-related human mitochondrial transcription factors B1 and B2 in mitochondrial biogenesis reveals new insight into maternally inherited deafness. Hum Mol Genet. 2009;18:2670-82.

24. Goto H, Tomono Y, Ajiro K, Kosako H, Fujita M, Sakurai M, et al. Identification of a novel phosphorylation site on histone $\mathrm{H} 3$ coupled with mitotic chromosome condensation. J Biol Chem. 1999;274:25543-9.

25. Van Hooser A, Goodrich DW, Allis CD, Brinkley BR, Mancini MA. Histone $\mathrm{H} 3$ phosphorylation is required for the initiation, but not maintenance, of mammalian chromosome condensation. J Cell Sci. 1998;111:3497-506.

26. Zhou Z, Hartwieg E, Horvitz HR. CED-1 is a transmembrane receptor that mediates cell corpse engulfment in C. elegans. Cell. 2001;104:43-56.

27. Li X, Johnson RW, Park D, Chin-Sang I, Chamberlin HM. Somatic gonad sheath cells and Eph receptor signaling promote germ-cell death in C. elegans. Cell Death Differ. 2012; 19:1080-9.

28. Gumienny TL, Lambie E, Hartwieg E, Horvitz HR, Hengartner MO. Genetic control of programmed cell death in the Caenorhabditis elegans hermaphrodite germline. Development. 1999;126:1011-22.

29. Michaelson D, Korta DZ, Capua Y, Hubbard EJA. Insulin signaling promotes germline proliferation in C. elegans. Development. 2010;137:671-80.

30. Dalfó D, Michaelson D, Hubbard EJA. Sensory regulation of reproduction via TGF $\beta$ signaling through the stem cell niche. Curr Biol. 2012;22:712-9.

31. Spike CA, Bader J, Reinke V, Strome S. DEPS-1 promotes Pgranule assembly and RNA interference in C. elegans germ cells. Development. 2008;135:983-93.

32. Sengupta MS, Low WY, Patterson JR, Kim H-M, Traven A, Beilharz TH, et al. ifet-1 is a broad-scale translational repressor required for normal $\mathrm{P}$ granule formation in $C$. elegans. J Cell Sci. 2013;126:850-9.
33. Wolke U, Jezuit EA, Priess JR. Actin-dependent cytoplasmic streaming in $C$. elegans oogenesis. Development. 2007; 134:2227-36.

34. Mishra P, Chan DC. Metabolic regulation of mitochondrial dynamics. J Cell Biol. 2016;212:379-87.

35. Liesa M, Shirihai Orian S. Mitochondrial dynamics in the regulation of nutrient utilization and energy expenditure. Cell Metab. 2013;17:491-506.

36. Youle RJ, van der Bliek AM. Mitochondrial fission, fusion, and stress. Science. 2012;337:1062-5.

37. Lee M-H, Ohmachi M, Arur S, Nayak S, Francis R, Church D, et al. Multiple Functions and Dynamic Activation of MPK-1 Extracellular Signal-Regulated Kinase Signaling in Caenorhabditis elegans Germline Development. Genetics. 2007;177:2039-62.

38. Francis R, Barton MK, Kimble J, Schedl T. gld-1, a tumor suppressor gene required for oocyte development in Caenorhabditis elegans. Genetics. 1995;139:579.

39. Kim S, Spike C, Greenstein D. Control of oocyte growth and meiotic maturation in C. elegans. Adv Exp Med Biol. 2013;757: https://doi.org/10.1007/978-1-4614-015-4_10.

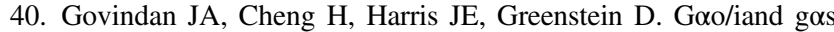
signaling function in parallel with the MSP/Eph receptor to control meiotic diapause in C. elegans. Curr Biol. 2006;16:1257-68.

41. Berg J, Hung YP, Yellen G. A genetically encoded fluorescent reporter of ATP:ADP ratio. Nat Methods. 2009;6:161-6.

42. Merritt C, Rasoloson D, Ko D, Seydoux G. 3'UTRs are the primary regulators of gene expression in the $\mathrm{C}$. elegans germline. Curr Biol. 2008;18:1476-82.

43. Oka M, Moriyama T, Asally M, Kawakami K, Yoneda Y. Differential role for transcription factor Oct4 nucleocytoplasmic dynamics in somatic cell reprogramming and self-renewal of embryonic stem cells. J Biol Chem. 2013;288:15085-97.

44. Morrison SJ, Spradling AC. Stem cells and niches: mechanisms that promote stem cell maintenance throughout life. Cell . 2008;132:598-611.

45. Joshi PM, Riddle MR, Djabrayan NJV, Rothman JH. C. elegans as a model for stem cell biology. Dev Dyn. 2010;239:1539-54.

46. Bratic I, Hench J, Henriksson J, Antebi A, Bürglin TR, Trifunovic A. Mitochondrial DNA level, but not active replicase, is essential for Caenorhabditis elegans development. Nucleic Acids Res. 2009;37:1817-28.

47. Kawasaki I, Hanazawa M, Gengyo-Ando K, Mitani S, Maruyama I, Iino Y. ASB-1, a germline-specific isoform of mitochondrial ATP synthase $b$ subunit, is required to maintain the rate of germline development in Caenorhabditis elegans. Mech Dev. 2007;124:237-51.

48. Arur S, Ohmachi M, Nayak S, Hayes M, Miranda A, Hay A, et al. Multiple ERK substrates execute single biological processes in Caenorhabditis elegans germ-line development. Proc Natl Acad Sci USA. 2009;106:4776-81.

49. Arur S, Ohmachi M, Berkseth M, Nayak S, Hansen D, Zarkower D, et al. MPK-1 ERK controls membrane organization in $C$. elegans oogenesis via a sex determination module. Dev Cell. 2011;20:677-88.

50. Miller MA, Nguyen VQ, Lee M-H, Kosinski M, Schedl T, Caprioli RM, et al. A sperm cytoskeletal protein that signals oocyte meiotic maturation and ovulation. Science. 2001;291:2144-7.

51. Kershner AM, Kimble J. Genome-wide analysis of mRNA targets for Caenorhabditis elegans FBF, a conserved stem cell regulator. Proc Natl Acad Sci USA. 2010;107:3936-41.

52. Crittenden SL, Bernstein DS, Bachorik JL, Thompson BE, Gallegos M, Petcherski AG, et al. A conserved RNA-binding protein controls germline stem cells in Caenorhabditis elegans. Nature. 2002;417:660-3.

53. Teixeira FK, Sanchez CG, Hurd TR, Seifert JRK, Czech B, Preall $\mathrm{JB}$, et al. ATP synthase promotes germ cell differentiation 
independent of oxidative phosphorylation. Nat Cell Biol. 2015;17:689-96.

54. Xu X, Duan S, Yi F, Ocampo A, Liu G-H, Izpisua Belmonte Juan C. Mitochondrial regulation in pluripotent stem cells. Cell Metab. 2013;18:325-32.

55. Cho YM, Kwon S, Pak YK, Seol HW, Choi YM, Park DJ, et al. Dynamic changes in mitochondrial biogenesis and antioxidant enzymes during the spontaneous differentiation of human embryonic stem cells. Biochem Biophys Res Commun. 2006;348:1472-8.

56. Zhang Y, Marsboom G, Toth PT, Rehman J. Mitochondrial respiration regulates adipogenic differentiation of human mesenchymal stem cells. PLoS ONE. 2013;8:e77077.

57. Varum S, Rodrigues AS, Moura MB, Momcilovic O, Easley CAIV, Ramalho-Santos J, et al. Energy metabolism in human pluripotent stem cells and their differentiated counterparts. PLoS ONE. 2011;6:e20914.
58. Armstrong L, Tilgner K, Saretzki G, Atkinson SP, Stojkovic M, Moreno R, et al. Human Induced pluripotent stem cell lines show stress defense mechanisms and mitochondrial regulation similar to those of human embryonic stem cells. Stem Cells. 2010;28:661-73.

59. Qin Z, Hubbard EJA. Non-autonomous DAF-16/FOXO activity antagonizes age-related loss of C. elegans germline stem/progenitor cells. Nat Commun. 2015;6:7107.

60. Korta DZ, Tuck S, Hubbard EJA. S6K links cell fate, cell cycle and nutrient response in $C$. elegans germline stem/progenitor cells. Development. 2012;139:859-70.

61. Syntichaki P, Troulinaki K, Tavernarakis N. eIF4E function in somatic cells modulates ageing in Caenorhabditis elegans. Nature. 2007;445:922-6.

62. Hansen M, Taubert S, Crawford D, Libina N, Lee S-J, Kenyon C. Lifespan extension by conditions that inhibit translation in Caenorhabditis elegans. Aging Cell. 2007;6:95-110. 